\title{
THE GOULD'S BELT DISTANCES SURVEY (GOBELINS). II. DISTANCES AND STRUCTURE TOWARD THE ORION MOLECULAR CLOUDS
}

\author{
Marina Kounkel $^{1}$, Lee Hartmann ${ }^{1}$, Laurent Loinard ${ }^{2,3}$, Gisela N. Ortiz-León ${ }^{2}$, Amy J. Mioduszewski ${ }^{4}$, \\ Luis F. Rodríguez ${ }^{2,5}$, Sergio A. Dzib ${ }^{3}$, Rosa M. Torres ${ }^{6}$, Gerardo PeCh $^{2}$, Phillip A. B. Galli ${ }^{7,8,9}$, Juana L. Rivera ${ }^{2}$, \\ Andrew F. Boden ${ }^{10}$, Neal J. Evans II $^{11}$, Cesar Briceño ${ }^{12}$, and John J. Tobin ${ }^{13,14}$ \\ ${ }^{1}$ Department of Astronomy, University of Michigan, 1085 S. University Street, Ann Arbor, MI 48109, USA; mkounkel@umich.edu \\ ${ }^{2}$ Instituto de Radioastronomía y Astrofísica, Universidad Nacional Autónoma de Mexico, Morelia 58089, Mexico \\ ${ }^{3}$ Max Planck Institut für Radioastronomie, Auf dem Hügel 69, D-53121 Bonn, Germany \\ ${ }^{4}$ National Radio Astronomy Observatory, Domenici Science Operations Center, 1003 Lopezville Road, Socorro, NM 87801, USA \\ ${ }_{5}$ King Abdulaziz University, P.O. Box 80203, Jeddah 21589, Saudi Arabia \\ ${ }^{6}$ Centro Universitario de Tonalá, Universidad de Guadalajara, Avenida Nuevo Perifrico No. 555, Ejido San José, Tatepozco, C.P. 48525, Tonalá, Jalisco, México \\ ${ }^{7}$ Université Grenoble Alpes, IPAG, F-38000, Grenoble, France \\ ${ }^{8}$ CNRS, IPAG, F-38000 Grenoble, France \\ ${ }^{9}$ Instituto de Astronomia, Geofísica e Ciências Atmosféricas, Universidade de São Paulo, Rua do Matão, 1226, \\ Cidade Universitária, 05508-900, São Paulo, Brazil \\ ${ }^{10}$ Division of Physics, Math and Astronomy, California Institute of Technology, 1200 East California Boulevard, Pasadena, CA 91125, USA \\ ${ }^{11}$ Department of Astronomy, The University of Texas at Austin, 2515 Speedway, Stop C1400, Austin, TX 78712-1205, USA \\ ${ }^{12}$ Cerro Tololo Interamerican Observatory, Casilla 603, La Serena, Chile \\ ${ }^{13}$ Homer L. Dodge Department of Physics and Astronomy, University of Oklahoma, 440 West Brooks Street, Norman, OK 73019, USA \\ ${ }^{14}$ Leiden Observatory, Leiden University, P.O. Box 9513, 2300-RA Leiden, The Netherlands \\ Received 2016 July 11; revised 2016 August 26; accepted 2016 September 13; published 2017 January 11
}

\begin{abstract}
We present the results of the Gould's Belt Distances Survey of young star-forming regions toward the Orion Molecular Cloud Complex. We detected 36 young stellar objects (YSOs) with the Very Large Baseline Array, 27 of which have been observed in at least three epochs over the course of two years. At least half of these YSOs belong to multiple systems. We obtained parallax and proper motions toward these stars to study the structure and kinematics of the Complex. We measured a distance of $388 \pm 5 \mathrm{pc}$ toward the Orion Nebula Cluster, $428 \pm 10 \mathrm{pc}$ toward the southern portion L1641, $388 \pm 10$ pc toward NGC 2068, and roughly 420 pc toward NGC 2024. Finally, we observed a strong degree of plasma radio scattering toward $\lambda$ Ori.
\end{abstract}

Key words: astrometry - ISM: individual objects (Orion Molecular Clouds) - parallaxes - radiation mechanisms: nonthermal - stars: kinematics and dynamics

Supporting material: machine-readable table

\section{INTRODUCTION}

Young star-forming regions toward Orion have been the subject of much interest, as they are the closest regions of a massive young stellar population. The star formation in the Orion Complex is concentrated in two molecular clouds, Orion $\mathrm{A}$ and $\mathrm{B}$, with clusters such as the Orion Nebula Cluster (ONC) and L1641 in Orion A, and NGC 2023/2024, NGC 2068/ 2072, and L1622 in Orion B. These clusters represent the most recent episodes of star formation in the region, which belong to the Orion $\mathrm{OB} 1 \mathrm{c}$ and $1 \mathrm{~d}$ subassociation, containing stars spanning ages from $\sim 1 \mathrm{Myr}$ up to $6 \mathrm{Myr}$ (Bally 2008). In addition to the clusters in the main cloud, other stellar groups in Orion host very young stars, like $\sigma$ Ori in the OB1b subassociation, and the groups of the $\lambda$ Ori association at the northernmost end of the complex.. Finally, a somewhat older (8-12 Myr) population is contained within the OB1a subassociation, where most of the parental gas has already been removed.

Over the course of the last century, many attempts have been made to measure distances to the Complex, particularly toward the ONC. Some of the earliest measurements were as high as $2000 \mathrm{pc}$ (Pickering 1917) and as low as $185 \mathrm{pc}$ (Kapteyn 1918). Eventually most measurements settled in the $350-500 \mathrm{pc}$ range and obtained through various means, most typically through zero-age main sequence fitting. Much of the scatter originated from inconsistent assumptions, models, and sample selection (see review by Muench et al. 2008).

For some time, the most widely used distance was $480 \pm 80 \mathrm{pc}$, obtained from proper motions of $\mathrm{H}_{2} \mathrm{O}$ masers toward the Orion $\mathrm{BN} / \mathrm{KL}$ region (Genzel et al. 1981). In the last decade, however, direct stellar parallax measurements of nonthermal emitting masers and stars were made possible through radio very long baseline interferometry (VLBI). Menten et al. (2007, hereafter MR) obtained a distance of $414 \pm 7 \mathrm{pc}$ from observations of four stars-GMR A, F, G, and 12-in the central (Trapezium) region of the ONC. Sandstrom et al. (2007, hereafter S07) also observed GMR A and obtained a somewhat closer distance of $389_{-21}^{+24} \mathrm{pc}$. Hirota et al. (2007) and Kim et al. (2008) observed $\mathrm{H}_{2} \mathrm{O}$ and $\mathrm{SiO}$ masers to obtain a distance of $437 \pm 19 \mathrm{pc}$ and $418 \pm 6 \mathrm{pc}$ respectively in the Orion $\mathrm{BN} / \mathrm{KL}$ region.

Other major efforts to measure a distance toward the ONC include Jeffries's (2007). He used stellar rotation to estimate distances of $440 \pm 34 \mathrm{pc}$ for his entire sample and $392 \pm 32 \mathrm{pc}$ including only stars without active accretion. Stassun et al. (2004) obtained a distance of $419 \pm 21 \mathrm{pc}$ through monitoring the kinematics of a double-line eclipsing binary system, assuming a value for the solar bolometric luminosity of $M_{\mathrm{bol}, \odot}=4.59$, although their distance estimate decreased to $390 \pm 21 \mathrm{pc}$ with $M_{\mathrm{bol}, \odot}=4.75$. Kraus et al. (2009) obtained a dynamical distance of $410 \pm 20 \mathrm{pc}$ based on 
modeling the orbit of the close binary $\theta^{1}$ Ori C. Some attempts have also been made to obtain distances from dust extinction maps not just toward the ONC, but also toward several distinct regions in the Orion Complex. Lombardi et al. (2011) estimated a distance of $371 \pm 10 \mathrm{pc}$ toward Orion $\mathrm{A}$ and $398 \pm 12$ pc toward Orion B using extinction maps measured from the Two-Micron All Sky Survey. Schlafly et al. (2014) provided distance estimates of 20 distinct regions through extinction from PanSTARRS photometry, although many of them are highly uncertain.

While the distance measured by MR is currently considered as canonical, it is based on a small sample of four stars. In addition, the MR stars all lie within the central regions of the ONC; however, the Complex spans $100 \mathrm{pc}$ projected on the sky, so it would not be surprising if the different regions of the cloud have substantially different distances, and it would not be surprising if the regions have differing radial distances of the same order. Therefore even if the distance toward the ONC is known with high accuracy, by applying this distance to other regions, an inherent uncertainty of $\sim 20 \%$, for example, could be introduced, as the Complex is located at the distance of $\sim 400 \mathrm{pc}$. This propagates to an error of $\sim 40 \%$ in luminosity, to $\sim 70 \%$ in the ages of young stars (Hartmann 2001).

Currently an ongoing mission of the Gaia space telescope is obtaining astrometry toward optically visible sources across the entire sky to measure parallaxes accurate to $100 \mu$ as for $G<17$ mag stars (de Bruijne et al. 2014), which should provide accuracy in distance measurements to within 5\%-10\% up to $1 \mathrm{kpc}$. VLBI observations can provide an important independent check on optical parallax measurements, as shown by the comparison of VLBI with Hipparcos distances for the Pleiades (Melis et al. 2014). In addition, radio VLBI can be useful for measuring sources in regions of high extinction and/ or significant nebulosity, as is the case in many regions of Orion.

In this paper we present radio VLBI observations of stellar parallaxes of young stellar objects (YSOs) identified toward the Orion Complex, hereby significantly expanding the number of stars in Orion with known distances and kinematics. This work is done as part of Gould's Belt Distances Survey (GOBELINS; Loinard et al. 2011), which is dedicated to measuring stellar parallaxes toward the Ophiuchus (Ortiz-León et al. 2017b), Serpens (Ortiz-León et al. 2017b), Taurus, Perseus, and Orion star-forming regions.

\section{OBSERVATIONS}

The observations presented in this paper were made with the National Radio Astronomy Observatory's Very Long Baseline Array (VLBA) at $5 \mathrm{GHz}$ with a $256 \mathrm{MHz}$ bandwidth (spanning the range of $4.852-5.076 \mathrm{GHz}$ ). They span a period of two years from 2014 to 2016 March, with observations preferentially scheduled near the equinoxes, to target the maxima of the parallactic eclipse along right ascension. All the fields were observed in groups of three per observing session, for a total of 56 observing sessions under the code of BL175 (Table 1). Each session was planned as follows: a primary calibrator was alternated between observing each field, and after five iterations encompassing all the fields, three secondary calibrators were observed. The duration of each pointing was $\sim 2$ minutes for targeted fields and $\sim 1$ minute for each of the calibrators. The geodetic block was observed at the beginning and the end of each session (the frequency of the observations of this block spanned the 4.596-5.076 GHz range). The total observing time was $\sim 1 \mathrm{hr}$ per field. In addition, we spent $1.5 \mathrm{hr}$ per session on the primary calibrator and $0.1 \mathrm{hr}$ on each of the secondary calibrators.

During correlation, each field was reduced to a series of small patches only a few arcseconds in diameter, and each patch was centered at a phase center corresponding to the targets within a field (a description of the process is presented in Ortiz-León et al. 2017a). Targets were identified from the Very Large Array (VLA) survey of the Orion Complex by Kounkel et al. (2014). In that survey 374 sources were detected, out of which 148 were associated with known YSOs and 86 were identified as YSO candidates on the basis of their radio properties out of the remaining sources that could not be classified by other means. The criteria for candidacy included (1) detectable circular polarization, or (2) short-term radio variability $>50 \%$ at either 4.5 or $7.5 \mathrm{GHz}$.

For the first two epochs of the VLBA Orion observations, 40 fields were observed (Table 1). The total number of fields was chosen on the basis of the number of hours awarded for GOBELINS. We accommodated observations of all five starforming clouds targeted by the program, and the field centers were distributed in such a way as to maximize the number of known YSOs observed. After two epochs, we were already able to begin to distinguish between galactic and extragalactic sources on the basis of motion of the sources between epochs. Twelve fields where no galactic sources have been detected were removed from the survey and six new fields were added to include more isolated YSOs from the VLA survey. The number of fields has been further cut to only 26 for epoch 4 , and to 17 for epoch 5 .

The data were reduced in the AIPS (Greisen 2003) following the standard prescription for the VLBA data. The multiband delays were removed using the DELZN task based on the geodetic sources (Reid \& Brunthaler 2004). The phase gradient across the sky was then calibrated using secondary calibrators with the ATMCA task. When multiple sources were observed in the same field, the same calibration was applied to all sources. Positions of all the sources were referenced to the primary calibrator. Finally, all sources were imaged and the positions of all point sources were extracted using the JMFIT task. More details on data reduction are presented in Ortiz-León et al. (2017a).

Positions of all sources were referenced relative to the primary calibrators (Table 2). The arrangement of the primary and secondary calibrators for each field is described in Table 3. This configuration was preserved through all the epochs. The exception to this were the $\lambda$ Ori fields, as their primary and secondary calibrators were extended, which resulted in uncertain astrometry. We propose that plasma scattering from the supernova bubble $2^{\circ} .5-3^{\circ}$ in radius around $\lambda$ Ori is responsible for the image blurring (see Appendix A). An attempt was made to switch to a different nearby calibrator that would improve the astrometry. However, as the assumed absolute positions of the calibrators are not referenced relative to each other, a positional offset was introduced to the sources in the field.

A few other calibrators do have some structure, most likely due to jet activity; however, an evolving jet structure should not significantly influence measured parallaxes (MR), particularly if the main source is point-like and the jet emission is sufficiently displaced. The two most notable calibrators with 
Table 1

Dates of the VLBA Observations

\begin{tabular}{|c|c|c|c|c|c|c|c|}
\hline$\#$ & $\begin{array}{l}\text { R.A. } \\
(\mathrm{J} 2000)\end{array}$ & $\begin{array}{l}\text { Decl. } \\
(\text { J2000) }\end{array}$ & $\begin{array}{c}\text { Epoch } 1 \\
\text { Date/Field Name }\end{array}$ & $\begin{array}{c}\text { Epoch } 2 \\
\text { Date/Field Name }\end{array}$ & $\begin{array}{c}\text { Epoch } 3 \\
\text { Date/Field Name }\end{array}$ & $\begin{array}{c}\text { Epoch } 4 \\
\text { Date/Field Name }\end{array}$ & $\begin{array}{c}\text { Epoch } 5 \\
\text { Date/Field Name }\end{array}$ \\
\hline 1 & $05: 35: 13.5550$ & $-05: 21: 37.8875$ & 03/24/14 GD1 & 09/21/14 EG1 & $02 / 26 / 15 \mathrm{FB} 1$ & 09/01/15 FP1 & $02 / 25 / 16$ FA1 \\
\hline 2 & 05:35:28.5199 & $-05: 10: 11.9953$ & $03 / 24 / 14$ GD2 & 09/21/14 EG2 & $02 / 26 / 15$ FB2 & 09/01/15 FP2 & $02 / 25 / 16$ FA2 \\
\hline 3 & 05:35:57.8954 & $-05: 23: 01.0259$ & 03/24/14 GD3 & 09/21/14 EG3 & $\cdots$ & $\cdots$ & $\cdots$ \\
\hline 4 & $05: 35: 32.0330$ & $-05: 39: 38.5578$ & $\cdots$ & $\cdots$ & $02 / 26 / 15$ FB3 & 09/01/15 FP3 & $\cdots$ \\
\hline 5 & $05: 34: 51.3847$ & $-04: 55: 18.2572$ & 03/10/14 GE1 & 09/22/14 EH1 & $02 / 27 / 15 \mathrm{FC} 2$ & 08/31/15 FQ2 & 03/01/16 HV2 \\
\hline 6 & $05: 34: 51.3847$ & $-05: 09: 09.6415$ & 03/10/14 GE2 & 09/22/14 EH2 & $02 / 27 / 15$ FC3 & 08/31/15 FQ3 & 03/01/16 HV3 \\
\hline 7 & 05:34:31.9858 & $-05: 28: 33.5795$ & 03/10/14 GE3 & 09/22/14 ЕH3 & $02 / 27 / 15 \mathrm{FCl}^{\mathrm{a}}$ & 08/31/15 FQ1 & 03/01/16 HV1 \\
\hline 8 & $05: 35: 49.5816$ & $-05: 16: 05.3335$ & 03/08/14 GF1 & 09/30/14 EI1 & 02/28/15 FD2 & 09/05/15 FR2 & 03/05/16 HW2 \\
\hline 9 & $05: 35: 59.2812$ & $-04: 56: 41.3956$ & 03/08/14 GF2 & 09/30/14 EI2 & $\cdots$ & $\cdots$ & $\cdots$ \\
\hline 10 & 05:36:08.9806 & $-05: 41: 01.8254$ & 03/08/14 GF3 & 09/30/14 EI3 & $02 / 28 / 15$ FD3 & 09/05/15 FR3 & 03/05/16 HW3 \\
\hline 11 & 05:35:02.4698 & $-05: 16: 05.3335$ & $\ldots$ & $\ldots$ & $02 / 28 / 15$ FD1 & $\ldots$ & $\ldots$ \\
\hline 12 & $05: 35: 16.3262$ & $-04: 41: 26.8728$ & 03/01/14 GG1 & 10/02/14 EJ1 & 03/01/15 FE1 & 09/13/15 FS1 & 03/08/16 HX1 \\
\hline 13 & $05: 35: 19.0975$ & $-04: 49: 45.7032$ & 03/01/14 GG2 & 10/02/14 EJ2 & 03/01/15 FE2 & 09/13/15 FS2 & 03/08/16 HX2 \\
\hline 14 & $05: 35: 46.8103$ & $-05: 29: 56.7182$ & 03/01/14 GG3 & 10/02/14 EJ3 & 03/01/15 FE3 & $\ldots$ & $\ldots$ \\
\hline 15 & 05:35:02.4698 & $-05: 34: 47.7026$ & 03/09/14 GH1 & 10/03/14 EK1 & 03/03/15 FF1 & 09/05/15 FR1 & 03/05/16 HW1 \\
\hline 16 & $05: 35: 21.8618$ & $-04: 29: 38.9299$ & 03/09/14 GH2 & 10/03/14 EK2 & $03 / 03 / 15 \mathrm{FF} 2$ & 09/13/15 FS3 & 03/08/16 HX3 \\
\hline 17 & 05:35:57.8954 & $-04: 40: 45.3036$ & 03/09/14 GH3 & 10/03/14 EK3 & 03/03/15 FF3 & $\cdots$ & $\cdots$ \\
\hline 18 & $05: 35: 45.4315$ & $-06: 16: 08.4821$ & 03/15/14 GI1 & 11/02/14 EL1 & 03/12/15 FG1 & $\cdots$ & $\cdots$ \\
\hline 19 & 05:36:21.4582 & $-06: 21: 41.0357$ & $03 / 15 / 14$ GI 2 & 11/02/14 EL2 & $03 / 12 / 15$ FG2 & 09/14/15 FT3 & $\ldots$ \\
\hline 20 & 05:37:52.9104 & $-06: 55: 37.9276$ & $03 / 15 / 14$ GI3 & 11/02/14 EL3 & $\ldots$ & $\ldots$ & $\cdots$ \\
\hline 21 & 05:40:25.3308 & $-08: 08: 22.6954$ & 03/02/14 GJ1 & 10/06/14 EM1 & $\ldots$ & $\ldots$ & \\
\hline 22 & $05: 41: 59.5543$ & $-08: 10: 27.4033$ & $03 / 02 / 14$ GJ2 & 10/06/14 EM2 & 03/06/15 FH1 & 09/14/15 FT1 & 03/10/16 HY1 \\
\hline 23 & $05: 42: 38.3525$ & $-08: 08: 22.6954$ & 03/02/14 GJ3 & 10/06/14 EM3 & 03/06/15 FH2 & $09 / 14 / 15$ FT2 & 03/10/16 HY2 \\
\hline 24 & $05: 41: 25.2326$ & $-02: 07: 02.2382$ & $03 / 23 / 14$ GK1 & $10 / 10 / 14$ EN1 & 03/08/15 FI1 & $\ldots$ & $\ldots$ \\
\hline 25 & 05:41:28.0039 & $-02: 21: 14.4072$ & $03 / 23 / 14$ GK2 & $10 / 10 / 14$ EN2 & $03 / 08 / 15$ FI2 & 09/15/15 FU1 & $\cdots$ \\
\hline 26 & 05:41:41.8603 & $-01: 54: 54.7769$ & $03 / 23 / 14$ GK3 & $10 / 10 / 14$ EN3 & $03 / 08 / 15$ FI3 & 09/15/15 FU2 & 03/12/16 HZ1 \\
\hline 27 & $05: 45: 39.6451$ & $-00: 08: 28.9269$ & 03/03/14 GL1 & 10/12/14 EO1 & 03/09/15 FJ1 & 09/19/15 FV1 & $\cdots$ \\
\hline 28 & 05:46:35.0707 & $+00: 04: 40.8882$ & 03/03/14 GL2 & 10/12/14 EO2 & 03/09/15 FJ2 & 09/19/15 FV2 & 03/12/16 HZ2 \\
\hline 29 & $05: 47: 05.5548$ & $+00: 21: 18.5494$ & 03/03/14 GL3 & 10/12/14 EO3 & 03/09/15 FJ3 & 09/19/15 FV3 & $\cdots$ \\
\hline 30 & 05:38:36.8107 & $-02: 35: 04.0477$ & 03/04/14 GM1 & 10/07/15 EP1 & $\cdots$ & $\cdots$ & $\cdots$ \\
\hline 31 & 05:39:15.6086 & $-02: 33: 40.9093$ & 03/04/14 GM2 & $10 / 07 / 15 \mathrm{EP} 2$ & $\cdots$ & $\cdots$ & $\cdots$ \\
\hline 32 & 05:40:56.1341 & $-02: 29: 33.2380$ & 03/04/14 GM3 & 10/07/15 EP3 & $\cdots$ & $\cdots$ & $\cdots$ \\
\hline 33 & 05:39:35.0076 & $-02: 41: 59.7401$ & $\cdots$ & $\cdots$ & 03/13/15 FK1 & 09/21/15 FW1 & 03/15/16 I01 \\
\hline 34 & $05: 41: 52.9454$ & $-02: 03: 55.1768$ & $\cdots$ & $\cdots$ & 03/13/15 FK2 & $09 / 21 / 15$ FW2 & 03/15/16 I02 \\
\hline 35 & $05: 34: 44.4564$ & $-04: 44: 54.7188$ & 03/06/14 GN1 & 10/14/14 EQ1 & $\ldots$ & $\ldots$ & $\ldots$ \\
\hline 36 & 05:35:02.4698 & $-06: 03: 12.0406$ & $03 / 06 / 14$ GN2 & 10/14/14 EQ2 & $\cdots$ & $\cdots$ & $\cdots$ \\
\hline 37 & 05:35:35.7252 & $-05: 52: 06.9330$ & $03 / 06 / 14$ GN3 & 10/14/14 EQ3 & $\cdots$ & $\cdots$ & $\cdots$ \\
\hline 38 & 05:34:39.9794 & $+10: 02: 14.5176$ & 03/16/14 GO1 & 10/19/14 ER1 & 03/14/15 FL1 & 09/28/15 FX1 & $\ldots$ \\
\hline 39 & $05: 35: 03.5354$ & $+09: 53: 34.9033$ & 03/16/14 GO2 & 10/19/14 ER2 & $03 / 14 / 15$ FL2 & $09 / 28 / 15$ FX2 & $\cdots$ \\
\hline 40 & 05:53:49.9392 & $+01: 37: 19.6637$ & 03/16/14 GO3 & 10/19/14 ER3 & $\cdots$ & $\cdots$ & $\ldots$ \\
\hline 41 & 05:46:01.8154 & $+00: 22: 41.6879$ & $\ldots$ & $\ldots$ & 03/14/15 FL2 & $\ldots$ & $\cdots$ \\
\hline 42 & 05:36:57.4848 & $-06: 52: 10.0816$ & 03/17/14 GP1 & 10/18/14 ES1 & $\cdots$ & $\ldots$ & $\cdots$ \\
\hline 43 & $05: 38: 48.3360$ & $-06: 59: 05.7736$ & $03 / 17 / 14$ GP2 & 10/18/14 ES2 & 03/16/15 FM2 & $\ldots$ & $\ldots$ \\
\hline 44 & 05:36:24.2294 & $-06: 45: 14.3892$ & $\cdots$ & $\cdots$ & 03/16/15 FM1 & $\cdots$ & $\cdots$ \\
\hline 45 & $05: 45: 47.9590$ & $+00: 14: 22.8572$ & 03/22/14 GQ1 & 10/21/14 ET1 & $\ldots$ & $\ldots$ & $\ldots$ \\
\hline 46 & 05:47:05.5548 & $+00: 12: 18.1495$ & 03/22/14 GQ2 & $10 / 21 / 14$ ET2 & $\cdots$ & $\ldots$ & $\ldots$ \\
\hline
\end{tabular}

Note.

${ }^{\mathrm{a}}$ Field was repositioned to R.A. $=05: 34: 34.7570$, decl. $=-05: 25: 47.3027$.

structure are J0539-0514 and J0532-0307. The former produced many errors in the calibration solutions as it was not bright enough during the first two epochs, with a typical flux of $\sim 30 \mathrm{mJy}$; the substructure was not immediately apparent, but during the third epoch its flux had increased to $\sim 50 \mathrm{mJy}$, resulting in a significant improvement in the calibration, and there was a clear emission from a second component at a position angle of $\sim 240^{\circ}, \sim 1$ mas away; this emission persisted during all the remaining epochs. The latter calibrator, J0532-0307, always had a spatially resolved second component at p.a. $\sim 150^{\circ}, \sim 10$ mas away.

Our VLBA survey fields covered a total of 300 sources from the VLA survey. As VLBA detections generally require nonthermal emission due to their high brightness temperatures, only 116 objects have been detected (although some of them can be resolved into multiple objects or a jetted structure). We report only on objects that either (1) were detected in at least two epochs, or (2) had a single detection $>5 \sigma$ (Figure 1 ). The 
Table 2

Assumed Positions of the Calibrators

\begin{tabular}{lcc}
\hline \hline Calibrator & $\begin{array}{c}\alpha \\
(\mathrm{J} 2000)\end{array}$ & $\begin{array}{c}\delta \\
(\mathrm{J} 2000)\end{array}$ \\
\hline J0539-0514 & $05: 39: 59.937192$ & $-05: 14: 41.30174$ \\
J0529-0519 & $05: 29: 53.533450$ & $-05: 19: 41.61678$ \\
J0541-0541 & $05: 41: 38.083371$ & $-05: 41: 49.42843$ \\
J0532-0307 & $05: 32: 07.519261$ & $-03: 07: 07.03799$ \\
J0517-0520 & $05: 17: 28.110157$ & $-05: 20: 40.84222$ \\
J0542-0913 & $05: 42: 55.877408$ & $-09: 13: 31.00660$ \\
J0553-0840 & $05: 53: 41.891558$ & $-08: 40: 01.90151$ \\
J0527-1002 & $05: 27: 24.060380$ & $-10: 02: 57.26651$ \\
J0558-0055 & $05: 58: 44.391460$ & $-00: 55: 06.92375$ \\
J0600-0005 & $06: 00: 03.503368$ & $-00: 05: 59.03477$ \\
J0552+0313 & $05: 52: 50.101499$ & $+03: 13: 27.24311$ \\
J0536+0944 & $05: 36: 31.978172$ & $+09: 44: 20.63128$ \\
J0532+0732 & $05: 32: 38.998486$ & $+07: 32: 43.34572$ \\
J0519+0848 & $05: 19: 10.811128$ & $+08: 48: 56.73450$ \\
J0544+1118 & $05: 44: 52.199795$ & $+11: 18: 49.92568$ \\
J0530+1331 & $05: 30: 56.416747$ & $+13: 31: 55.14954$ \\
J0547+1223 & $05: 47: 06.276323$ & $+12: 23: 46.24477$ \\
\hline
\end{tabular}

Note.When imaged, secondary calibrators are offset from these positions as their coordinates are referenced to the primary calibrator.

remaining undetected objects most likely emit only thermally. Thirty-six of the detected systems can be definitely identified as YSOs on the basis of astrometric motion, and 57 are most likely associated with active galactic nucleus (AGN) activity. The remaining 23 objects have detection in only one epoch or have astrometry too poor to make a definitive determination (Table 4).

Out of 148 objects in the VLA survey that were known YSOs in the literature, 36 have been detected with VLBA. Three of these sources have been falsely identified, as we cannot confirm their membership in the Orion Complex on the basis of their astrometry. GBS-VLA J054121.69-021108.3 (=VLBA 55), GBS-VLA J053542.27-051559.3 (=VLBA 110), and GBS-VLA J053532.03-053938.6 (=VLBA 139) all previously identified as YSOs on the basis of optical and IR emission, but they do not show a significant astrometric offset between epochs.

Kounkel et al. (2014) identified 86 VLA sources as candidate YSOs, based on their radio properties, and we detected 26 of them with the VLBA. Only three of these can be confirmed as YSOs in the Orion region. The criteria for selection used by Kounkel et al. (2014) appear to be not entirely reliable: while a strong variability at $4.5 \mathrm{GHz}$ can indeed be used to distinguish galactic from extragalactic sources, the same cannot be said for $7.5 \mathrm{GHz}$ (Figure 2). Similarly, the degree by which circular polarization can be affected by beam squint has been significantly underestimated. Out of nine sources with observed VLA circular polarization detected in this program, we can confirm only two as YSOs.

\section{FITTING}

To fit the parallax and the proper motions, the IDL routine MPFIT (Markwardt 2009) was used. This routine fits a given model to data by minimizing least-squares fit. At the end of each iteration, it outputs only a single array with the weighted differences between the data and the model, and any number of equations can be solved simultaneously.

For a single object, the motion of a star in the plane of the sky is prescribed by

$$
\begin{gathered}
\alpha(t)=\alpha_{0}+\mu_{\alpha} \cos \delta t+\pi f_{\alpha}(\alpha, \delta, t) \\
\delta(t)=\delta_{0}+\mu_{\delta} t+\pi f_{\delta}(\alpha, \delta, t),
\end{gathered}
$$

where $\alpha_{0}$ and $\delta_{0}$ are positions of the star at a given reference time, and $\mu_{\alpha}$ and $\mu_{\delta}$ are the components of the proper motion. In addition, $f_{\alpha}$ and $f_{\delta}$ are the projections over $\alpha$ and $\delta$ of the parallactic eclipse, and they are given by (e.g., Seidelmann 1992)

$$
\begin{gathered}
f_{\alpha}=(X \sin \alpha-Y \cos \alpha) / \cos \delta \\
f_{\delta}=X \cos \alpha \sin \delta+Y \sin \alpha \sin \delta-Z \cos \delta,
\end{gathered}
$$

where $X, Y$, and $Z$ are the barycentric coordinates of the Earth in units of au, tabulated using the Python package Skyfield. ${ }^{15}$

The uncertainties in the fitted parameters are twofold. First, they depend on the positional uncertainties of all the individual detections of the stars as measured by JMFIT, driven by the resolution of VLBA and the flux of the object. This does not take into account possible various systematic offsets in positions between different epochs, which could be significantly larger than nominally quoted positional uncertainties. Typically, the estimation of errors due to systematic offsets is usually done by examining the goodness of the parallactic fit and scaling positional uncertainties until the reduced $\chi^{2}$ of the fit becomes equal to 1 (e.g., MR; S07).

Approximately half of GK main-sequence stars and $30 \%$ of M stars belong to multiple systems (Duquennoy \& Mayor 1991; Fischer \& Marcy 1992; Raghavan et al. 2010; Duchêne \& Kraus 2013), and the motion of the binary projected onto the plane of the sky can degrade the goodness of the fit. These multiple systems can be roughly divided into three categories, based on the effect they have on parallax and proper motion fit.

1. Binaries with orbital periods much longer than the total monitoring time covered by this program (e.g., $\gg 10$ years). As the star would only barely move in its orbit, this motion would be approximately linear. It is possible to introduce and fit for an acceleration term to correct for the minor shifts due to nonlinearity. Determination of the parallax should not be affected by these binaries. Proper motion would not represent the true proper motion of the system, as it is strongly affected by the orbital motion of the star.

2. Binaries with intermediate orbital periods. The effects of the binary motion cannot be ignored during the parallactic fit due to the noticeably changing acceleration of the star; therefore, it is necessary to fit the Keplerian parameters for the binary and the parallax simultaneously. The main orbital parameters are the semimajor axis $a_{1}$ of the primary, the orbital period $P$, the eccentricity $e$, the argument of the pericenter $\omega$, the time of passage of the pericenter $T_{P}$, the inclination $i$, the argument of the ascending node $\Omega$, and in case of the astrometric binary with both components detected, the mass ratio $q$. The mean anomalies for the dates of observations are calculated with a given $P$ and $T_{P}$. Then a true anomaly $\theta$ and a radius from the center of mass $r$ are determined

\footnotetext{
15 http://rhodesmill.org/skyfield/
} 
Table 3

Calibrator Setup for the Observed Fields

\begin{tabular}{|c|c|c|c|c|}
\hline $\begin{array}{l}\text { Field } \\
\#\end{array}$ & Primary & $\begin{array}{c}\text { Secondary } \\
1\end{array}$ & $\begin{array}{c}\text { Secondary } \\
2\end{array}$ & $\begin{array}{c}\text { Secondary } \\
3\end{array}$ \\
\hline $1,2,3,4,8,9,10,11,12,13,14,15,16,17$ & J0539-0514 & J0529-0519 & J0541-0541 & J0532-0307 \\
\hline $5,6,7,35,36$ & J0529-0519 & J0539-0514 & J0532-0307 & J0517-0520 \\
\hline $18,19,20,37$ & J0539-0514 & J0541-0541 & J0529-0519 & J0542-0913 \\
\hline $21,22,23$ & J0542-0913 & J0541-0541 & $\mathrm{J} 0553-0840$ & J0527-1002 \\
\hline $24,25,26$ & J0532-0307 & J0539-0514 & J0541-0541 & J0558-0055 \\
\hline $27,28,29,45,46$ & J0558-0055 & J0600-0005 & $\mathrm{J} 0552+0313$ & J0532-0307 \\
\hline $30,31,32,33,34$ & J0532-0307 & J0539-0514 & J0529-0519 & J0558-0055 \\
\hline 38,39 & J0536+0944 & $\mathrm{J} 0532+0732$ & $\mathrm{~J} 0544+1118$ & $\mathrm{~J} 0530+1331$ \\
\hline 40 & $\mathrm{~J} 0552+0313$ & J0600-0005 & J0558-0055 & J0606-0024 \\
\hline 41 & $\mathrm{~J} 0552+0313$ & J0532-0307 & J0558-0055 & J0606-0024 \\
\hline $42,43,44$ & $\mathrm{~J} 0541-0541$ & J0539-0514 & J0529-0519 & J0542-0913 \\
\hline
\end{tabular}

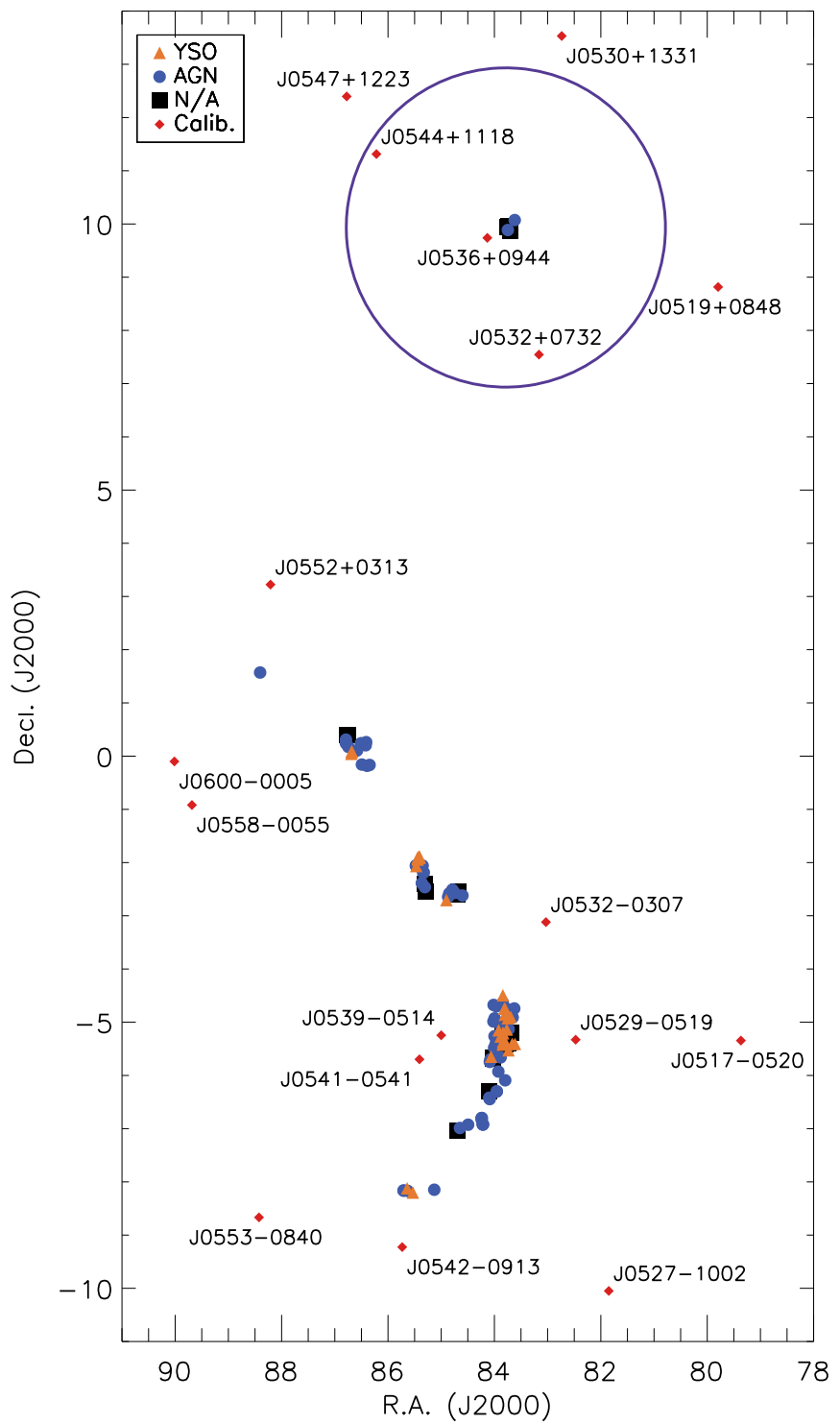

Figure 1. Locations of the sources observed by this program. They are separated into the confirmed YSOs, sources that are most likely associated with AGN activity, and the ones that could not be identified as either due to an insufficient number of epochs observed or to poor astrometry. Locations of all the calibrators are also included with their names. The circle at the top of the figure is $3^{\circ}$ in radius, and it shows the approximate position of the $\lambda$ Ori ring. along a Keplerian orbit with a given $e$ for the positions corresponding to these mean anomalies. This orbit is scaled and projected onto the plane of the sky through

$$
\begin{gathered}
\alpha(t)=a_{1} r(\cos (\theta+\omega) \sin \Omega \\
-\sin (\theta+\omega) \cos \Omega \cos i) / \cos \delta \\
\delta(t)=a_{1} r(\sin (\theta+\omega) \sin \Omega \cos i+\cos (\theta+\omega) \cos \Omega) .
\end{gathered}
$$

For secondary stars, $\theta$ is rotated by $180^{\circ}$, and $a_{2}$ is used instead, which is scaled from $a_{1}$ by $q$. The fitting code is not optimized for determining several of the Keplerian parameters in a robust manner, as MPFIT is not a global optimizer and can get stuck in the local minima if the initial guesses for the parameters are not optimal. For this reason, we explore a parameter grid of the initial guesses of $P$ in steps of 0.2 years, $e$ in steps of $0.1, T_{P}$ in steps of $P / 12$, and $\omega$ in steps of $30^{\circ}$. The final values of these parameters can be fine-tuned by the code, and the remaining orbital and parallactic parameters are fitted directly. The uncertainties are determined from the combination of the uncertainties produced by the fits as well as the scatter in the fits from the various initial guesses for the parameters in the grid. Due to a limited number of available epochs, there is a minor dependency in some of the fitted parameters (e.g., parallax and proper motions) between the different realizations of the fits, although the exact trend between the fitted distance and proper motions may be more or less systematic depending on any number of factors, e.g., number of epochs monitored, inclination, or any other physical properties of the system (Figure 3), although the uncertainties in the parameters do take the range of scatter into account. The results of these fits are typically comparable within $1 \sigma$ to the fits produced by the Binary Star Combined Solution Package from Gudehus (2001). The comparison of these two implementations of the binary fitting algorithm is discussed in Ortiz-León et al. (2017a).

3. Binaries with an orbital period smaller than the time between the consecutive epochs of observation (e.g., $<6$ months). As the stars in these compact binaries should not move far from their center of mass, the overall fit should approximate that of a single star but with somewhat larger uncertainties in the parallax due to the random sampling of the positions of the star in its orbit, and the effect becomes minimal with a sufficiently large number of epochs. If the star in question belongs to a known spectroscopic binary with a constrained 
Table 4

Positions of Sources Identified in at Least Two Epochs or with a Single $>5 \sigma$ Detection

\begin{tabular}{|c|c|c|c|c|c|c|}
\hline VLBA & YSO? & $\begin{array}{c}\text { R.A. } \\
\text { (J2000, mas) }\end{array}$ & $\begin{array}{c}\text { Decl. } \\
\text { (J2000, mas) }\end{array}$ & $\begin{array}{l}\text { Date } \\
\text { (JD) }\end{array}$ & $\begin{array}{l}\text { Flux } \\
(\mathrm{mJy})\end{array}$ & $\begin{array}{c}\text { Field rms } \\
(\mathrm{mJy})\end{array}$ \\
\hline 1 & $\mathrm{n}$ & $5: 35: 29.221269 \pm 0.361$ & $-5: 05: 44.137403 \pm 0.491$ & 2456741 & $0.593 \pm 0.171$ & 0.074 \\
\hline 1 & $\mathrm{n}$ & $5: 35: 29.221299 \pm 0.157$ & $-5: 05: 44.136384 \pm 0.402$ & 2456922 & $0.570 \pm 0.174$ & 0.072 \\
\hline 1 & $\mathrm{n}$ & $5: 35: 29.221309 \pm 0.945$ & $-5: 05: 44.136624 \pm 0.418$ & 2457266 & $1.695 \pm 0.488$ & 0.079 \\
\hline 1 & $\mathrm{n}$ & $5: 35: 29.221302 \pm 0.254$ & $-5: 05: 44.136877 \pm 0.485$ & 2457444 & $0.702 \pm 0.192$ & 0.052 \\
\hline
\end{tabular}

Note. The uncertainties for both $\alpha$ and $\delta$ are given in units of mas.

(This table is available in its entirety in machine-readable form.)
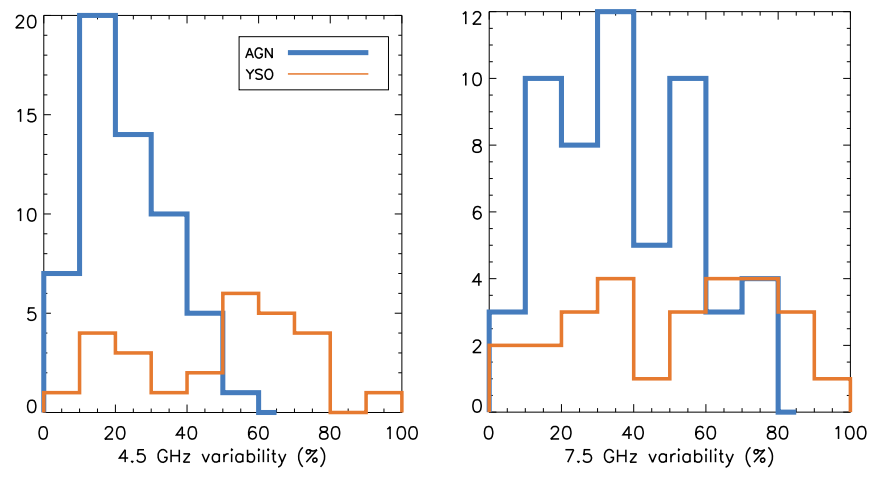

Figure 2. Variability reported by Kounkel et al. (2014) for all sources that can be distinguished as galactic and extragalactic on the basis of VLBA astrometry.

orbit, then by superimposing the orbit onto the parallactic motion it is possible to minimize this offset and determine a more reliable distance through fitting of the inclination $i$ and the longitude of the ascending node $\Omega$ for the system. In this case $e, P, a_{1} \sin i, T_{P}, \omega$, and $q$ are held fixed to the known values from the spectroscopic orbital solutions.

\section{DISCUSSION}

Twenty stars have been detected toward the ONC, and we present distance solutions to 15 of them. Six of these stars belong to the Trapezium cluster. Two stars have been detected toward L1641, three toward NGC 2068, and one toward $\sigma$ Ori. Seven stars have been detected toward NGC 2024, and we present preliminary distance solutions to five of them. These solutions are presented in Table 5 and discussed in Appendices $\mathrm{B}$ for $\mathrm{ONC}$ and $\mathrm{C}$ for the remaining regions. The individual fits are presented in Figure 4.

Three stars are found to be astrometric binaries with both components detected: VLBA 4/107, 27/28, and 61/62. We presently can fit for the orbital motion of VLBA 4/107 and 61/ 62 , the parameters of which are presented in Table 6. Another two, VLBA 58 and 68, are also identified as belonging to multiple systems with an intermediate period on the basis of their astrometry, although only a single star has been detected. In addition, VLBA 125, 126, and 145 have been detected in only three epochs, but they also show possible signatures of multiplicity. Six stars-VLBA 6, 9, 11, 19, 27, and 34-are known spectroscopic binaries with short periods. The distance solution of VLBA 11, 19, and 34 incorporates the orbital motion. The parameters of the orbit are presented in Table 7 . Another four stars-VLBA 4/107, 8, 11, and 27/28-have also either known or identified long-period companions in addition to the aforementioned nearby companions (with the exception of VLBA 8, they belong to high-order multiple systems).

\subsection{Revised Distance to the Trapezium}

Out of six stars observed in the Trapezium, four have been previously observed by MR, although those authors have incorporated only three of them into the distance solution for the cluster. A simultaneous parallactic fit of all the stars found toward the Trapezium in this program results in a distance of $383 \pm 3$ pc. This fit is produced by fitting the equations of motions of all the stars at the same time with a single distance but different proper motions for all stars. The result of this fit is also identical to the weighted average of the individual measurements. The weighted average distance of all the stars in the ONC is $388 \pm 2 \mathrm{pc}$. These values do not include possible systematic effects due to the phase gradient (see below). This is discrepant with the distance of $414 \pm 7 \mathrm{pc}$ obtained by MR by $3 \sigma$, or $\sim 0.2$ mas in parallax. It is possible that there is a systematic offset of such magnitude between different epochs that cannot be reproduced during fitting by merely scaling the positional uncertainties until the $\chi^{2}$ of the fit is equal to 1 , so it is possible that the formal uncertainties are somewhat underestimated. And since all of these stars are observed in a single field, any systematic offset that is applied to the coordinates of the center of the field will be propagated to the positions of the sources, and the parallaxes and proper motions could be affected accordingly. However performing a fit with a reduced number of epochs in either work done by MR or this work offers no reconciliation, so the effect is not dominated by a pointing error in any single epoch.

Low-frequency radio observations could be affected by the dispersive delay (e.g., Reid \& Honma 2014), which is difficult to calibrate, resulting in a phase gradient across the sky and producing a slight offset in the absolute positions of the targeted objects. This effect becomes large the farther the object is from the primary calibrator. The effect does average out with the sufficiently large number of epochs; nonetheless, some of it can propagate to the parallax estimation. This effect can lead to somewhat different distances when using different calibrators as a reference for the absolute coordinates of the targeted sources.

To estimate the strength of this phase gradient, we compare the distances of the Trapezium sources with the coordinates referenced to the observed positions of the secondary calibrators (Figure 1). The primary calibrator for the field is J0539-0514, which is located 1.2 away from the targets. The simultaneous fit gives $394 \pm 3 \mathrm{pc}$ when all the coordinates are referenced to J0529-0519 (1.3 away), $375 \pm 3$ pc relative to J0541-0541 (1.6 away), and $382 \pm 3 \mathrm{pc}$ relative to $\mathrm{J} 0532-0307$ ( 2.4 away). As the Trapezium sources are located approximately halfway 

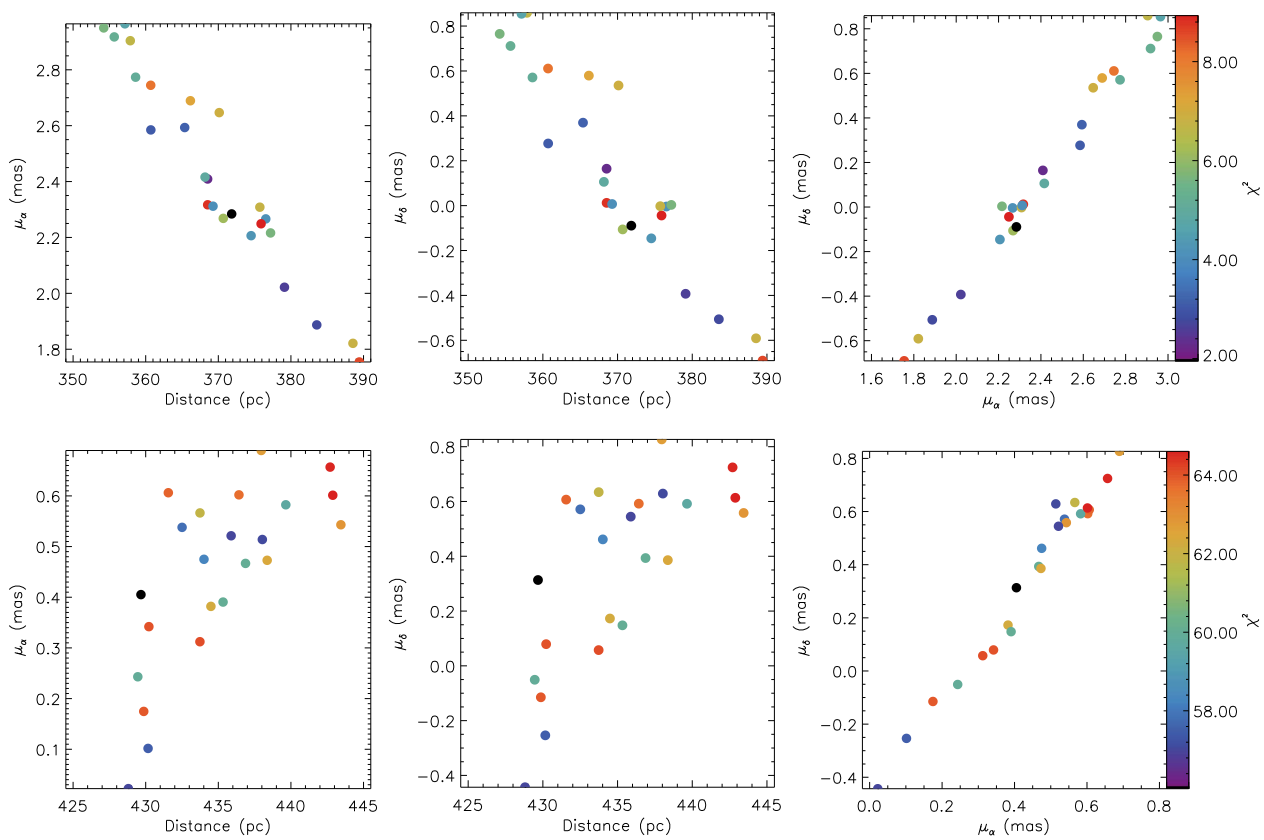

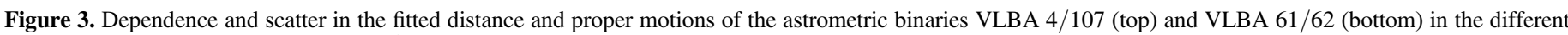
realization of the fit up to the reduced $\chi^{2}$ of 10 and 65 , respectively.

between J0539-0514 and J0529-0519, we can estimate the systematic effect of the dispersive delay on the parallax toward it to be on the order of 0.033 mas ( $5 \mathrm{pc}$ at the distance of the ONC). This effect is consistent throughout the ONC as long as these calibrators are separated by less than 1.5 from the target. Referencing the coordinates to J0529-0519 tends to produce somewhat larger distances than referencing them to J0539-0514. This is also true in the cases where J0529-0519 is used as the primary calibrator (i.e., in the cases of VLBA 14, 16, 18, and 19). We add $5 \mathrm{pc}$ (or 0.033 mas) in quadrature to the uncertainties in the weighted average distance to account for this systematic effect. We adopt a distance of $388 \pm 5$ pc toward the ONC, including the Trapezium.

This analysis cannot be performed on the solutions obtained by MR, as they have observed only a single calibrator. On the one hand, as their observations were obtained at a higher frequency $(8.4 \mathrm{GHz})$, they should be less susceptible to the dispersive delay. On the other hand, they have obtained fewer epochs, so this effect is somewhat more likely to propagate to the parallax and their primary calibrator is somewhat farther away.

Some minor differences could also be attributed to the difference in the fitting routines. Both the codes used in this work and work done by MR produce fits within $1 \sigma$ of each other when applied to a particular set of positions. However, MR assume a circular orbit for the Earth and fit parallax only from $\alpha$, using $\delta$ only to fit $\mu_{\delta}$, whereas the code used in this work considers the effect of the parallax on both $\alpha$ and $\delta$ using the true orbit of the Earth. Fitting the positions quoted in MR produces a combined distance of $406 \pm 4$ pc including GMR $\mathrm{G}$, or a distance of $409 \pm 3$ pc excluding it.

While it is possible to make a single fit for each star utilizing the data obtained by both $\mathrm{S} 07, \mathrm{MR}$, and this work, the difficulty lies in the fact that each survey utilized a different observing and calibration strategy. S07 used J0541-0541 as a primary calibrator and J0529-0519 as the secondary calibrator. MR used J0541-0541 as the primary calibrator as well as the geodetic sources. This work used J0539-0514 as the primary calibrator with three secondary calibrators and geodetic sources. The absolute positions of the calibrators that are found in common between these works are assumed to be somewhat different, up to a $\sim 1$ mas level. All of these factors introduce an offset in the absolute positions between these works that is not found in observing the sources repeatedly with the same calibration strategy. While it is possible to calibrate the magnitude of this offset, the exact fit is strongly dependent on the manner of calibration, so it is best to treat the data obtained by different projects independently. However, as both this work and S07 have a larger number of observations than the work done by MR, the fit utilizing all the positions does seem to favor a significantly closer distance than the one found by MR.

Multiplicity is another possible culprit of the difference between the fits. At least two stars observed by MR and used in their distance estimate are spectroscopic binaries, which makes distance solutions produced by them inherently more uncertain. We analyzed the effect that these orbits could have on the distance. The effect on the distance of GMR 12 is within $1 \mathrm{pc}$. No orbital solution currently exists for GMR F. For the remaining sources observed by this program and identified as spectroscopic binaries, the effect varies between 3 and $18 \mathrm{pc}$. While it is possible that the effect can contribute to the difference between these two works, it is unlikely that it could explain the systematic nature of the offset. However, it must be noted that the orbital motion parameters can be greatly affected by any systematic offsets in the data, particularly when the orbits cannot be fitted a priori.

Nonetheless, there is sufficient evidence to suggest that the ONC is located closer than has been previously estimated by MR. Systematic offsets would affect each pointing differently. Therefore, a larger number of fields with a larger number of epochs and a larger number of sources overall through the ONC implies that the overall effect of the systematic offsets, if it is present, would be more noticeable 
Table 5

Distance and Proper Motion Solutions

\begin{tabular}{|c|c|c|c|c|c|c|c|}
\hline VLBA & $\begin{array}{l}\text { Common } \\
\text { Names }\end{array}$ & $\begin{array}{l}\text { Spectral } \\
\text { Type }^{\mathrm{e}}\end{array}$ & Region & $\begin{array}{l}\text { Parallax } \\
\text { (mas) }\end{array}$ & $\begin{array}{l}\text { Distance } \\
(\mathrm{pc})\end{array}$ & $\begin{array}{c}\mu_{\alpha} \\
\left(\operatorname{mas~yr}^{-1}\right)\end{array}$ & 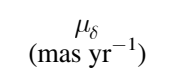 \\
\hline 5 & GMR A & & Trapezium & $2.673 \pm 0.112$ & $374 \pm 15$ & $1.81 \pm 0.11$ & $\overline{-1.62 \pm 0.13}$ \\
\hline $6^{\mathrm{a}}$ & GMR F & K1IV (H97) & Trapezium & $2.567 \pm 0.051$ & $389 \pm 7$ & $2.38 \pm 0.08$ & $0.55 \pm 0.14$ \\
\hline 8 & GMR G & K4III (H97) & Trapezium & $2.646 \pm 0.041$ & $377 \pm 5$ & $3.82 \pm 0.10$ & $1.60 \pm 0.17$ \\
\hline $9^{\mathrm{a}}$ & $\theta^{1}$ Ori E & G2IV (M12) & Trapezium & $2.557 \pm 0.051$ & $391 \pm 7$ & $1.45 \pm 0.03$ & $1.02 \pm 0.08$ \\
\hline $11^{\mathrm{a}}$ & $\theta^{1}$ Ori A & B0V (H97) & Trapezium & $2.626 \pm 0.100$ & $380 \pm 14$ & $4.81 \pm 0.10$ & $-2.53 \pm 0.12$ \\
\hline 14 & V1699 Ori & & ONC & $2.493 \pm 0.049$ & $401 \pm 7$ & $1.76 \pm 0.05$ & $-0.89 \pm 0.16$ \\
\hline $16^{\mathrm{b}}$ & Parenago 1469 & G9IV (H97) & ONC & $2.533 \pm 0.027$ & $394 \pm 4$ & $-7.22 \pm 0.06$ & $-0.99 \pm 0.08$ \\
\hline 18 & Parenago 1724 & K0III (S09) & ONC & $2.509 \pm 0.044$ & $398 \pm 7$ & $0.06 \pm 0.20$ & $6.95 \pm 0.16$ \\
\hline $19^{\mathrm{a}}$ & Parenago 1540 & $\mathrm{~K} 3 \mathrm{~V}+\mathrm{K} 5 \mathrm{~V}(\mathrm{M} 88)$ & ONC & $2.591 \pm 0.046$ & $385 \pm 6$ & $-4.01 \pm 0.08$ & $-1.17 \pm 0.07$ \\
\hline 22 & HD 37150 & B3III (H99) & ONC & $2.536 \pm 0.046$ & $394 \pm 7$ & $1.32 \pm 0.05$ & $-0.56 \pm 0.12$ \\
\hline $34^{\mathrm{a}}$ & HD 37017 & B2V (H99) & ONC & $2.643 \pm 0.075$ & $378 \pm 10$ & $1.88 \pm 0.09$ & $1.20 \pm 0.14$ \\
\hline $58^{\mathrm{c}}$ & & & NGC 2024 & $2.223 \pm 0.121$ & $449 \pm 24$ & $0.04 \pm 0.31$ & $0.20 \pm 0.43$ \\
\hline $61 / 62^{c}$ & & & NGC 2024 & $2.306 \pm 0.054$ & $434 \pm 10$ & $0.47 \pm 0.32$ & $0.39 \pm 0.62$ \\
\hline $125^{\mathrm{b}, \mathrm{d}}$ & & & NGC 2024 & $1.865 \pm 0.105$ & $536 \pm 30$ & $-0.43 \pm 0.16$ & $1.03 \pm 0.42$ \\
\hline $126^{\mathrm{b}, \mathrm{d}}$ & & & NGC 2024 & $2.804 \pm 0.032$ & $356 \pm 4$ & $0.55 \pm 0.10$ & $-0.10 \pm 0.15$ \\
\hline $148^{\mathrm{b}}$ & V621 Ori & & NGC 2024 & $2.422 \pm 0.034$ & $412 \pm 5$ & $0.19 \pm 0.44$ & $-0.97 \pm 0.27$ \\
\hline $145^{\mathrm{b}, \mathrm{d}}$ & HD 294300 & G5 (A00) & $\sigma$ Ori & $3.303 \pm 0.353$ & $302 \pm 32$ & $-4.92 \pm 0.66$ & $4.67 \pm 1.37$ \\
\hline 63 & & & NGC 2068 & $2.608 \pm 0.047$ & $383 \pm 7$ & $-1.02 \pm 0.10$ & $-0.52 \pm 0.15$ \\
\hline $68^{c}$ & HD 290862 & B3 (S75) & NGC 2068 & $2.197 \pm 0.545$ & $455 \pm 113$ & $0.35 \pm 0.27$ & $0.83 \pm 0.83$ \\
\hline 69 & & A0 (S75) & NGC 2068 & $2.547 \pm 0.034$ & $392 \pm 5$ & $0.01 \pm 0.10$ & $-0.49 \pm 0.08$ \\
\hline
\end{tabular}

Notes.

a Spectroscopic binary.

b Detected only in three epochs. Uncertainties could be underestimated.

c Astrometric binary.

d Probable binary.

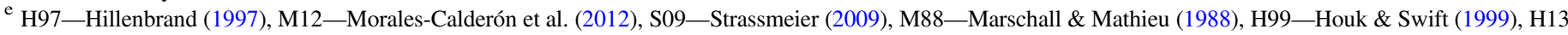
—Hsu et al. (2013), A00—Alcalá et al. (2000), S75—Strom et al. (1975).

in this work than in the work done by MR. The consistency in both the fitted distance and the proper motion estimates of GMR A between this work and that done by S07 suggests that our results are reproducible, which would also be less likely if there was a significant positional offset in our data. Finally, the consistency in distance toward GMR G between this work and that done by MR is surprising, given the lack of consistency between other sources.

Finally, there exists the curious case of the NGC 1980 cluster, which is located south of Trapezium in the vicinity of $\iota$ Ori. This cluster has a somewhat older population of stars compared to the rest of ONC (4-5 Myr; Alves \& Bouy 2012). Unfortunately we do not detect any YSOs toward it, but it is thought to be located at the distance of $380 \mathrm{pc}$ obtained through pre-main-sequence fitting (Bouy et al. 2014). This is why this cluster was thought to exist in the foreground of ONC as a separate entity. Nonetheless, the kinematics of NGC 1980 do not show any unique features not present in $\mathrm{ONC}$, and in fact, the velocity dispersion toward it is the smallest of any other region found toward the ONC (Da Rio et al. 2016; Kounkel et al. 2016). If we assume a significantly closer distance toward the Trapezium and the ONC than what was previously assumed by MR, then NGC 1980 should not be considered a foreground cluster but rather an integral part of the ONC.

\subsection{Structure of the Orion Complex}

The weighted average distance with the weighted uncertainty of all the stars located toward the ONC, including the Trapezium, is $388 \pm 5 \mathrm{pc}$. The distance measurements to nearly all stars is consistent with the average distance for the cluster (Figure 5). There is some scatter in the individual measurements of distance. Most of this scatter is likely to be systematic in nature. Some of this scatter may be physical, as the stars detected toward the ONC span a $4 \mathrm{pc}$ region in the plane of the sky at the distance of $388 \mathrm{pc}$. It is also possible that some substructure is present in the ONC; however, due to limited sampling this possibility is not definitive.

The southern end of L1641 appears to be located considerably farther away, at $428 \pm 10 \mathrm{pc}$ (Figure 6). We include the effect of the dispersive delay in this value, which we estimate to be comparable to what we found in the ONC. Unfortunately we cannot measure it directly, as all of the secondary calibrators are located too far away from the targeted YSOs for their positions to be useful. 

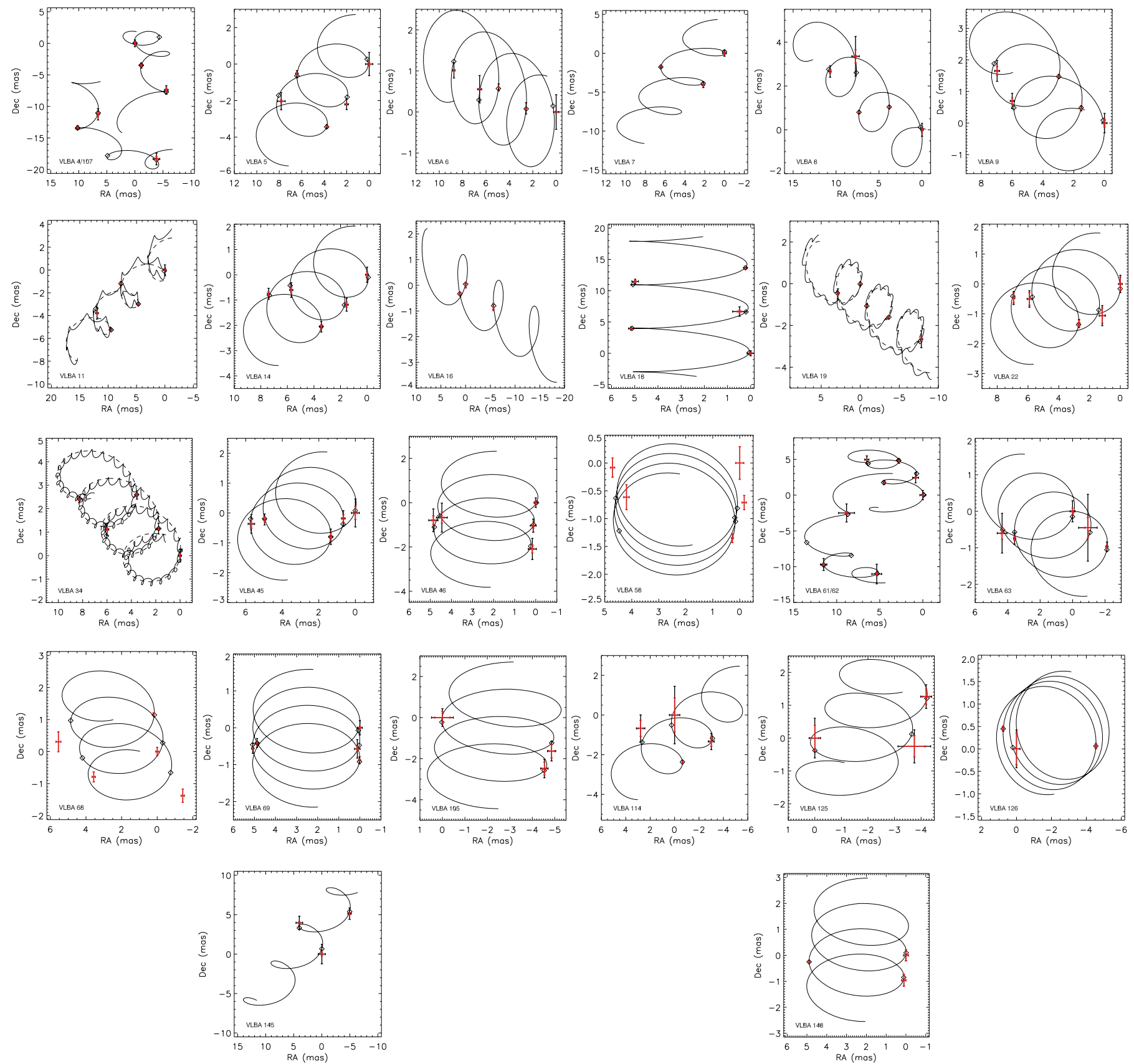

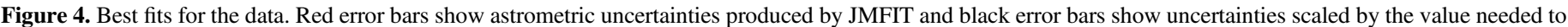

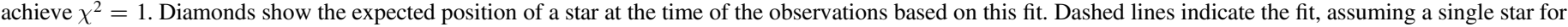
spectroscopic binaries.

The exact manner in which L1641 connects to the ONC is unclear as there are no galactic sources detected in the northern part of the filament. However, it is not unreasonable to assume that the northern part of the L1641 filament should be located at a similar distance to the ONC. There is a smooth gradient in radial velocity (RV) along the Orion A molecular cloud, ranging from $\sim 8$ to $4 \mathrm{~km} \mathrm{~s}^{-1}$ from the northern to the southern end of the L1641 (Bally et al. 1987; Nishimura et al. 2015). This could imply either a passive rotation of the cloud, moving from a very inclined to a more face-on orientation, or it could be the result of something actively pushing on the gas and causing it to accelerate (it is notable that the Orion Complex lies near the edge of the Orion-Eridanus superbubble; Ochsendorf et al. 2015). If the latter is true, it could potentially explain a large number of stars in the ONC that appear to be blueshifted relative to the molecular gas (Kounkel et al. 2016), as their RV would be representative of the initial velocity of the gas rather than the current velocity.

It is difficult to determine how accurate our measurement of the distance toward NGC 2068 is, as all of the calibrators, including the primary calibrator, are more than $3^{\circ}$ away. We estimate the effect of the dispersive delay to be on the order of 0.066 mas or $10 \mathrm{pc}$ at the distance of NGC 2068. We find a distance to NGC 2068 of $388 \pm 10$ pc. Finally, a distance toward NGC 2024 at this time cannot be reliably measured, given the high incidence of multiplicity as well as a limited number of observations currently available for the stars found toward this region. Neither can we currently obtain a reliable 
Table 6

Orbital Solutions for Astrometric Binaries

\begin{tabular}{lcc}
\hline \hline Parameters & VLBA 61/62 & VLBA 4/107 \\
\hline$P$ (year) & $9.50 \pm 0.67$ & $6.27 \pm 0.54$ \\
$T_{p}(\mathrm{HJD})$ & $2456455 \pm 60$ & $2457355 \pm 200$ \\
$e$ & $0.50 \pm 0.12$ & $0.40 \pm 0.02$ \\
$\omega(\mathrm{deg})$ & $92 \pm 20$ & $268 \pm 7$ \\
$q=M_{2} / M_{1}$ & $0.50 \pm 0.26$ & $0.99 \pm 0.24$ \\
$a_{1}(\mathrm{au})$ & $2.10 \pm 0.51$ & $(2.63 \pm 0.17) / \cos i$ \\
$a_{2}(\mathrm{au})$ & $4.19 \pm 0.70$ & $(2.53 \pm 0.42) / \cos ^{3}$ \\
$M_{1}\left(M_{\odot}\right)$ & $1.85 \pm 0.58$ & $(1.70 \pm 0.16) / \cos ^{3} i$ \\
$M_{2}\left(M_{\odot}\right)$ & $0.95 \pm 0.22$ & $(1.62 \pm 0.38) / \cos ^{3} i$ \\
$i(\mathrm{deg})$ & $141 \pm 6$ & $\ldots$ \\
$\Omega($ deg $)$ & $122 \pm 30$ & 3 \\
\hline$N_{\text {obs }}($ primary) & 5 & 3 \\
$N_{\text {obs }}($ secondary $)$ & 3 & \\
\hline
\end{tabular}

distance toward the $\sigma$ Ori cluster. This would be resolved with further monitoring. At this time, we estimate the distance toward NGC 2024 on the basis of measurements toward VLBA $61 / 62$ and VLBA 148 to be $423 \pm 15$ pc.

\subsection{Proper Motions and Runaway Stars}

Parallax and proper-motion solutions $\left(v_{\mathrm{pm}}\right)$ are available for 15 systems in the ONC. While this is insufficient to perform a detailed analysis of the kinematics of the region, it is possible to obtain mean motions of the cluster.

Proper motions of long-period binaries (i.e., VLBA 8, 9, 11, and $4 / 107)$ are not representative of the $v_{\mathrm{pm}}$ of the cluster, as they are significantly affected by the orbital motion. Therefore, we do not include them in the calculation of the mean. Three stars can be considered significant outliers in terms of proper motions. VLBA 16,18 , and 19 have a $v_{\mathrm{pm}}$ range of between 4.2 and $7.3 \mathrm{mas} \mathrm{yr}^{-1}\left(7.7-13.4 \mathrm{~km} \mathrm{~s}^{-1}\right)$. The difference is much larger than the typical dispersion velocity of $2.5 \mathrm{~km} \mathrm{~s}^{-1}$ found toward the ONC (Kounkel et al. 2016), so they are also not included in the calculation of the proper motion of the cluster. The remaining seven stars suggest a $v_{\mathrm{pm}}$ for the ONC of $\mu_{\alpha}^{\mathrm{lsr}}=1.35 \pm 0.70 \mathrm{mas} \mathrm{yr}^{-1}=2.49 \pm 1.29 \mathrm{~km} \mathrm{~s}^{-1}$ and $\mu_{\delta}^{\mathrm{lsr}}=$ $-1.44 \pm 1.51 \mathrm{mas} \mathrm{yr}^{-1}=-2.66 \pm 2.79 \mathrm{~km} \mathrm{~s}^{-1}$, in the local standard of rest reference frame, corrected for the peculiar motion of the Sun (Figure 7). The uncertainties are obtained from the variance in the individual measurement, although they could be somewhat overestimated as the variance is largely driven by the peculiar velocity of stars within a cluster.

The most likely explanation for the high $v_{\mathrm{pm}}$ for VLBA 16 , 18 , and 19 is that they have been ejected from the cluster through a dynamical interaction within the cluster core, with the most notable suspect being $\theta^{1}$ Ori $C$. Unfortunately this star has not been detected by this program, but assuming that its proper motion should be similar to the average proper motion of the cluster, all three runaway stars appear to originate in its vicinity. Assuming linear motion, VLBA 16 appears to have been ejected $\sim 8 \times 10^{4}$ year ago, VLBA $18 \sim 12 \times 10^{4}$ year ago, and VLBA $19 \sim 8 \times 10^{4}$ year ago. Some deceleration probably has occurred as they moved through the cluster; however, assuming the potential calculated by Hillenbrand \& Hartmann (1998), this deceleration is not significant compared to the current $v_{\mathrm{pm}}$ of these stars.
VLBA 16, 18, and 19 are not alone in suffering the fate of being runaway stars. Additionally, Poveda et al. (2005) identified JW 451 and 349 as runaways from $\theta^{1}$ Ori C; however this was later disproven by O'Dell et al. (2005). More famously, sources $\mathrm{BN}, \mathrm{I}$, and $n$ in the Orion $\mathrm{BN} / \mathrm{KL}$ nebula have been accelerated to speeds of up to $26 \mathrm{~km} \mathrm{~s}^{-1}$ through a dynamical interaction 500 yr ago (Gómez et al. 2008; Goddi et al. 2011). In an even more extreme case, $\mu \mathrm{Col}$, AE Aur, and the compact binary $\iota$ Ori are thought to be ejected from the Trapezium cluster some $2.5 \mathrm{Myr}$ ago through a four-body interaction (de Zeeuw et al. 2001; Gualandris et al. 2004).

The average proper motion of L1641 is $\mu_{\alpha}^{\text {lsr }}=0.82 \pm$ $0.39 \mathrm{mas} \mathrm{yr}^{-1}=1.67 \pm 0.79 \mathrm{~km} \mathrm{~s}^{-1}$ and $\mu_{\delta}^{\mathrm{lsr}}=-2.20 \pm$ $0.38 \mathrm{mas} \mathrm{yr}^{-1}=-4.48 \pm 0.78 \mathrm{~km} \mathrm{~s}^{-1}$. The southern end of the cloud appears to move westward relative to the $\mathrm{ONC}$, although it is not collapsing into the ONC directly (Figure 8).

Analysis of the proper motions of NGC 2024 is once again made more complex by the multiplicity of the sources. The motions of VLBA 58, 125, and 126, if they are indeed binaries, would be at least partially affected by the orbital motion. We obtained an orbital fit for VLBA 61/62, but the proper motions remain rather uncertain. Finally, VLBA 148 appears to be moving away from the cluster, and its motion appears to be rather distinct from the uncertain motions of the other stars. It is possible that it could have been ejected through dynamical interactions in the cluster, although further monitoring would be necessary to confirm this (Figure 9).

The proper motion of NGC 2068 based on VLBA 63 and 69 is $\mu_{\alpha}^{\text {lsr }}=-0.62 \pm 0.73 \mathrm{mas} \mathrm{yr}^{-1}=-1.15 \pm 1.34 \mathrm{~km} \mathrm{~s}^{-1}$ and $\quad \mu_{\delta}^{\text {lsr }}=-1.27 \pm 0.02$ mas yr$^{-1}=-2.35 \pm 0.04 \mathrm{~km} \mathrm{~s}^{-1}$ (Figure 10). It appears to move toward NGC 2024, although this does not take into account the relative velocities of the two clusters.

It is clear that the entire complex appears to move in the southern direction on the equatorial globe, or in the direction of galactic rotation (Figure 11). Orion A also moves preferentially toward the East (toward the galactic plane), and the ONC has the largest eastward velocity compared to the other regions of the complex.

\section{CONCLUSIONS}

We monitored 36 nonthermal radio-emitting YSOs spread throughout the Orion Complex with VLBA over a period of two years, and we report measured stellar parallaxes toward 26 of them. Fifteen are located toward the ONC, and we find a distance of $388 \pm 5 \mathrm{pc}$ to the cluster; this is somewhat closer than the canonical $414 \pm 7 \mathrm{pc}$ distance found by MR that is typically used in the literature. This result has implications for the luminosity and ages of the cluster. If the cluster is $7 \%$ closer than previous estimations, then this implies that it is $12 \%$ fainter and 20\% older (assuming a relation $t \propto L^{-3 / 2}$ ) than what was previously reported in surveys of the ONC such as the one by Da Rio et al. (2010).

We also report distances toward other regions located in the Orion Complex, such as L1641, NGC 2024, and NGC 2068. While these values are somewhat more uncertain due to a significantly smaller sample size, limited spatial coverage (particularly in case of L1641), and multiplicity, these are the first direct measurements of the stellar parallaxes toward these regions. This provides insight into the structure of the Complex.

We identify a possible region of a large degree of plasma scattering toward the $\lambda$ Ori star-forming region. The degree of 
Table 7

Orbital Solutions for Spectroscopic Binaries

\begin{tabular}{lccr}
\hline \hline Parameters & VLBA 11 & VLBA 19 & VLBA 34 \\
\hline$P$ (days) & $65.4314491 \pm 0.0028542$ & $33.73 \pm 0.030$ & $18.6561 \pm 0.0002$ \\
$T_{p}$ (HJD) & $2444195.5773 \pm 0.4426$ & $2444972.95 \pm 1.75$ & $2446010.461 \pm 0.080$ \\
$e$ & $0.6261 \pm 0.0313$ & $0.12 \pm 0.01$ & $0.468 \pm 0.014$ \\
$\omega($ deg $)$ & $183.2838 \pm 5.1861$ & $131.3 \pm 6.7$ & $118.3 \pm 2.4$ \\
$a_{1} \sin i(\mathrm{au})$ & $0.16 \pm 0.01$ & $0.081 \pm 0.002$ & $0.069 \pm 0.003$ \\
$a_{2} \sin i(\mathrm{au})$ & $\ldots$ & $0.107 \pm 0.003$ & $0.132 \pm 0.009$ \\
Reference & Stickland $\&$ Lloyd (2000) & Marschall \& Mathieu (1988) & Bolton et al. (1998) \\
\hline$i($ deg $)$ & $87 \pm 11$ & $104 \pm 12$ & $52 \pm 23$ \\
$\Omega($ deg $)$ & $150 \pm 20$ & $61 \pm 11$ & $313 \pm 26$ \\
$M_{1}\left(M_{\odot}\right)$ & $\ldots$ & $0.49 \pm 0.10$ & $4.09 \pm 4.44$ \\
$M_{2}\left(M_{\odot}\right)$ & $\ldots$ & $0.37 \pm 0.07$ & $2.14 \pm 2.22$ \\
\hline
\end{tabular}

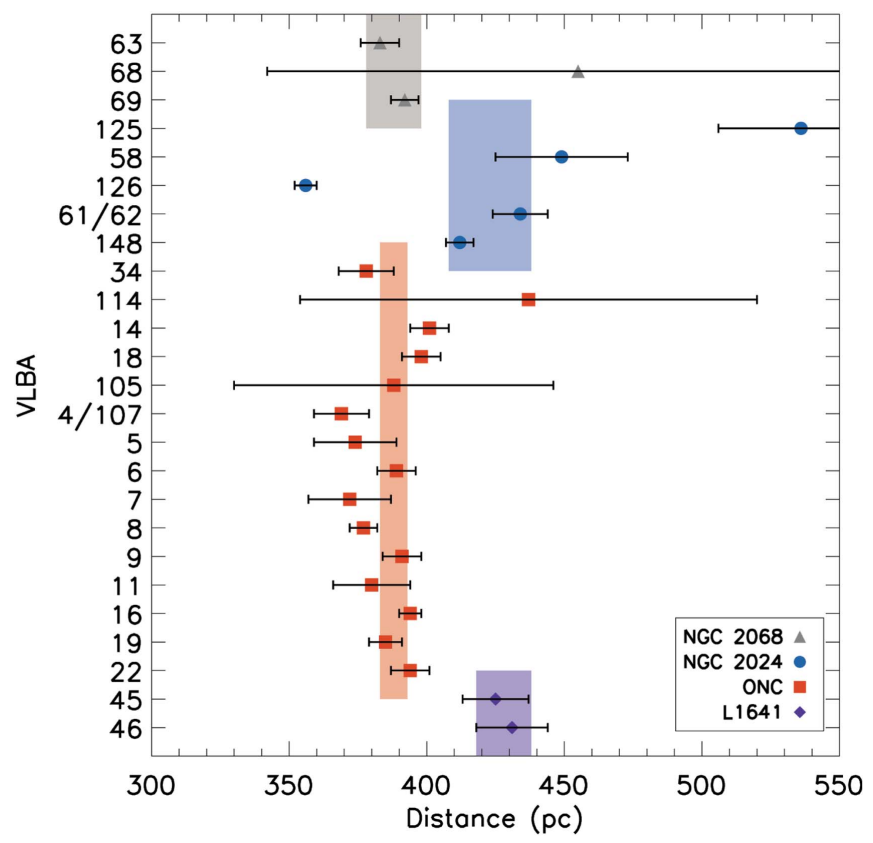

Figure 5. Measured distances to the individual stars in the four clusters, sorted according to their $\delta$. The averages for each cluster are shown with semitransparent rectangles.

scattering is significant, with broadening of the observed size of the objects of up to 16.5 mas near the center of the cluster at $5 \mathrm{GHz}$. The scattering is spread all within the ring $2^{\circ} .5-3^{\circ}$ in radius produced by supernova activity. Unfortunately, this effect made it impossible to measure the astrometry accurately enough to obtain a parallax toward the stars found in this region.

A persistent problem in the analysis of both the parallax and proper motions of the stars is the multiplicity. We conclusively identify five of 27 stars that have been detected in at least three epochs belonging to a multiple system with orbital periods between six months and 10 years, with at least three more systems identified as likely binaries, although further monitoring would be necessary to confirm them. It is impossible to accurately determine parallaxes to these systems without solving for the orbital motion of these systems, which we can presently do for only two of them. Six stars are known spectroscopic binaries with very short periods (one of them also has an aforementioned intermediate-period companion); a larger number of them could have very close companions that

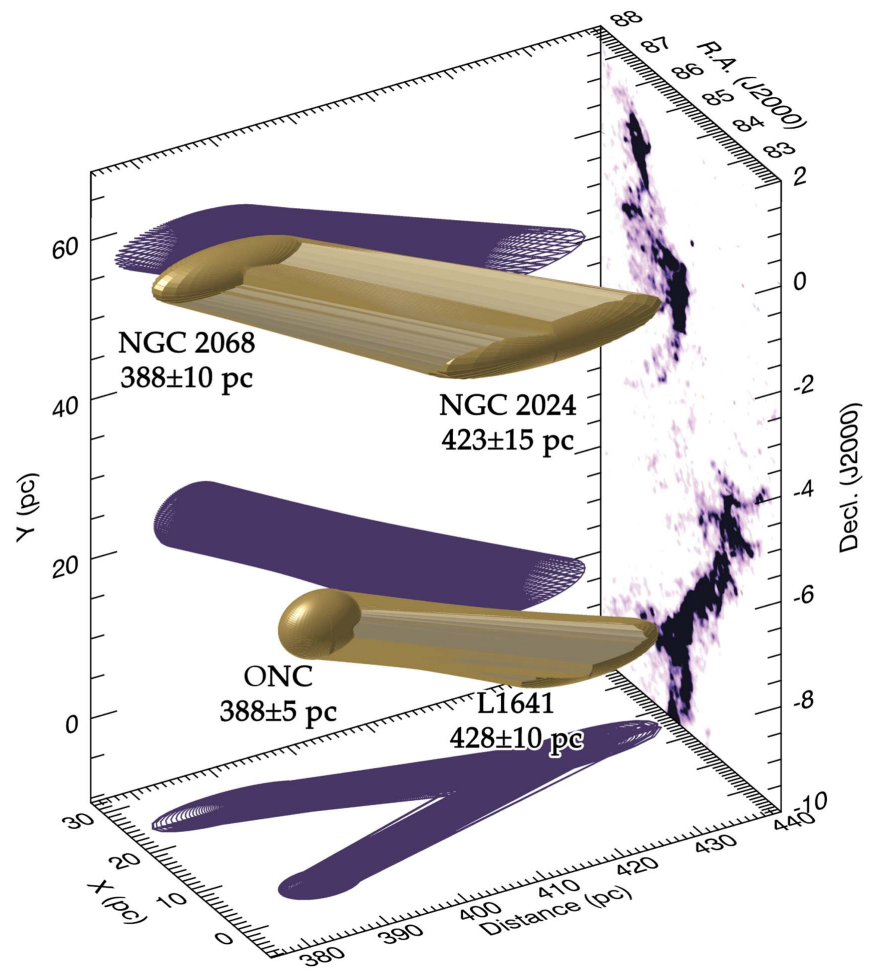

Figure 6. 3D model of the Orion Complex. The width of the end ellipsoids in the model along the distance is representative of the uncertainties in the measurement and not the actual depth of each cluster. The plane of the sky plane shows the extinction map from Gutermuth et al. (2011). Purple shadows are the projections of the model onto the remaining planes. Conversion of the length along the plane of the sky to pc is done at the distance of $388 \mathrm{pc}$.

are yet to be identified, particularly since few surveys of spectroscopic binaries have been performed in the Orion Complex outside of the ONC. While an understanding of their orbits is not detrimental to finding the parallax, it could still influence the solution somewhat. Finally, four stars in the sample have long-period companions, although only one of them does not have a closer companion in a higher-order multiple system. These wide companions should not affect the solution for the parallax, although they do affect proper motions.

In all, at least 14 (possibly more) of 27 stars observed with VLBA belong to multiple systems. Whether this multiplicity fraction is consistent with that for the entire Complex is not yet known as it is difficult to identify companions with 


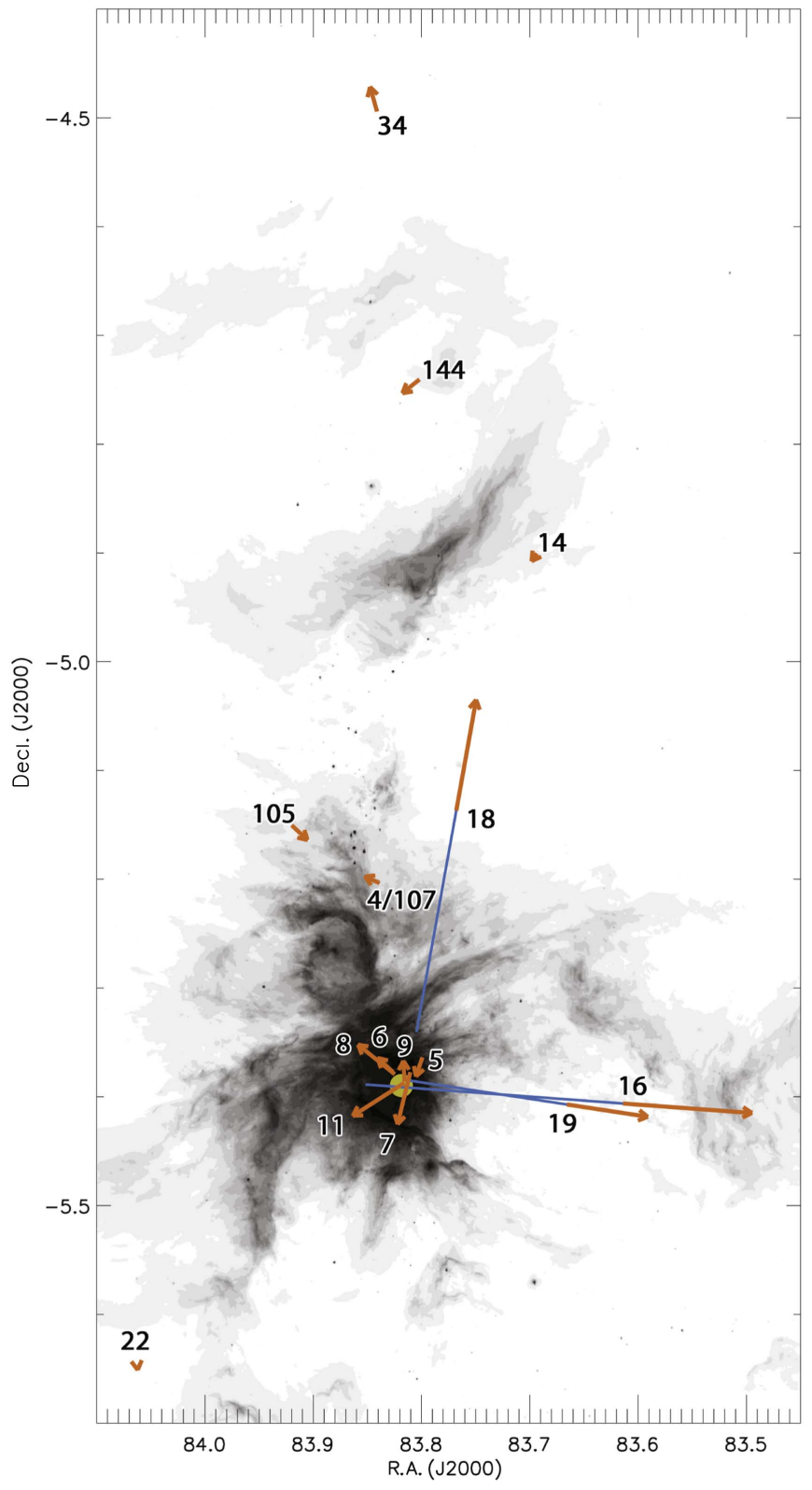

Figure 7. Proper-motion vectors of the stars detected toward the ONC,

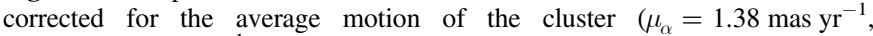

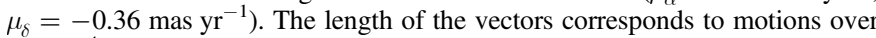
$5 \times 10^{4}$ years. The yellow dot at the center shows the current position of $\theta^{1}$ Ori C. Blue lines show the trajectory of the runaway stars over the last $10^{5}$ years. All the sources are labeled with their VLBA number. The grayscale background is the $8 \mu \mathrm{m}$ Spitzer map from Megeath et al. (2012).

intermediate periods toward Orion due to its distance. Future generations of high-resolution optical and IR telescopes would make it possible to identify the full extent of multiplicity toward this region.

Further monitoring of the identified YSOs would be beneficial: due to variability in radio, only a limited number of detections are available to some stars. In the future, it will be possible to more effectively measure their parallax and proper motions. It will also be necessary to confirm multiplicity and constrain orbital parameters toward some sources.

The distance solutions produced by the GOBELINS survey will be used as an independent constraint on the accuracy of

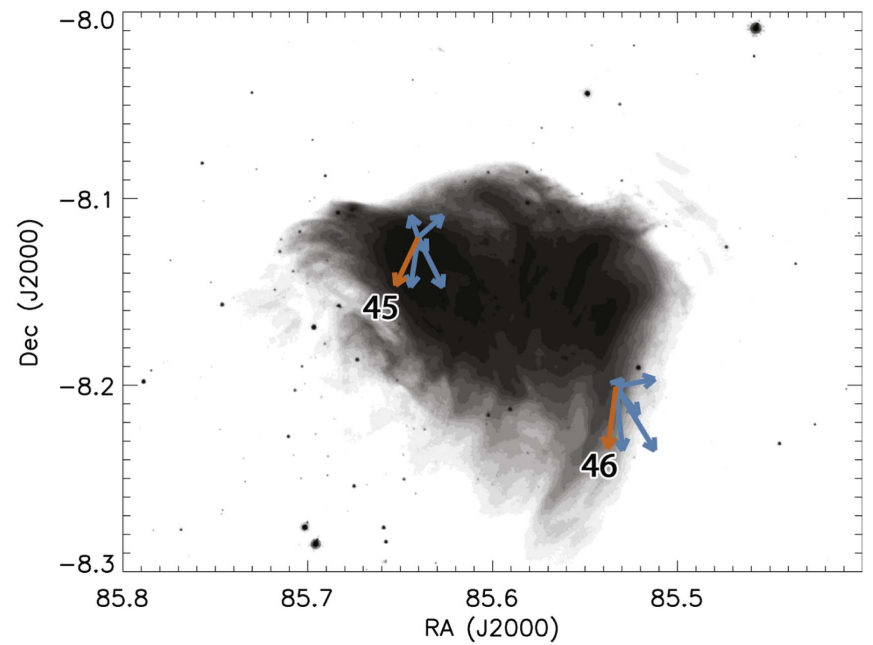

Figure 8. Proper motion vectors of the stars detected toward L1641 in the local standard of rest reference frame. The length of the vectors corresponds to motions over $5 \times 10^{4}$ years. Orange vectors are the measured proper motions, blue vectors are motions relative to the average motion of the ONC with a combination of $\pm 1 \sigma$ formal uncertainty of the average motion of the ONC in both $\mu_{\alpha}$ and $\mu_{\delta}$. All the sources are labeled with their VLBA number. The grayscale background is $8 \mu \mathrm{m}$ Spitzer map from Megeath et al. (2012).

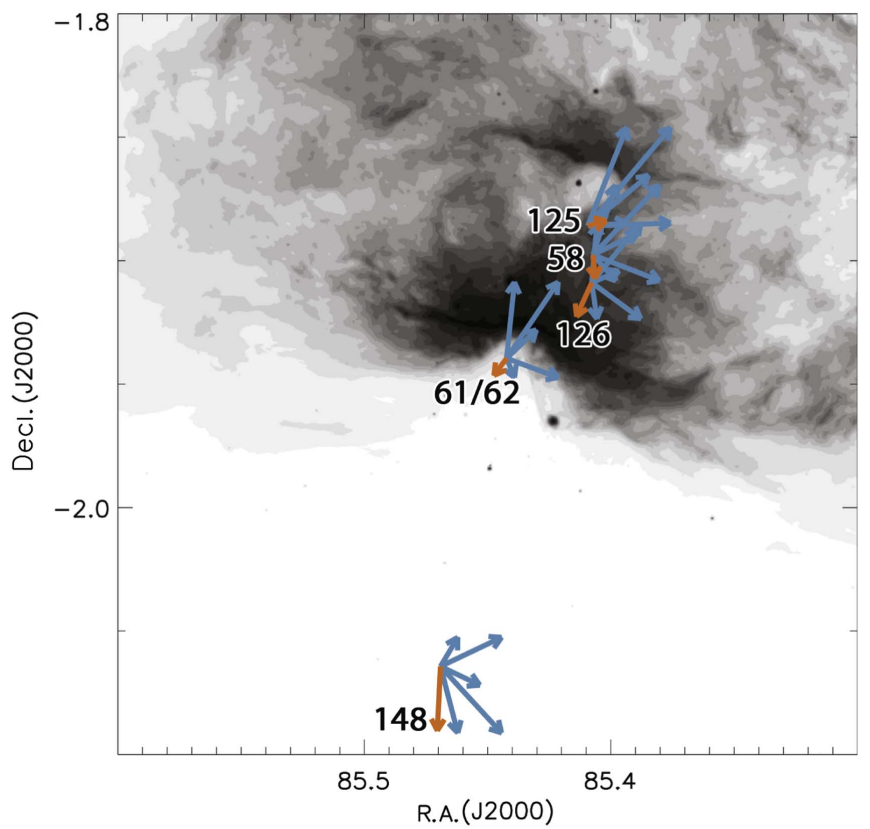

Figure 9. Same as Figure 8, but for NGC 2024.

Gaia, as the systematic effects behind the sample selection and the individual observations are different between these two programs. Approximately half of the systems observed with VLBA toward the Orion Complex are optically visible, so it should be possible to compare the distance solutions toward them directly, at least in the ONC, although nebulosity could significantly degrade performance in the optical regime. However, star-forming regions toward Orion B suffer from high extinction; therefore only a few members of NGC 2024 and NGC 2068 would be detectable with Gaia. 


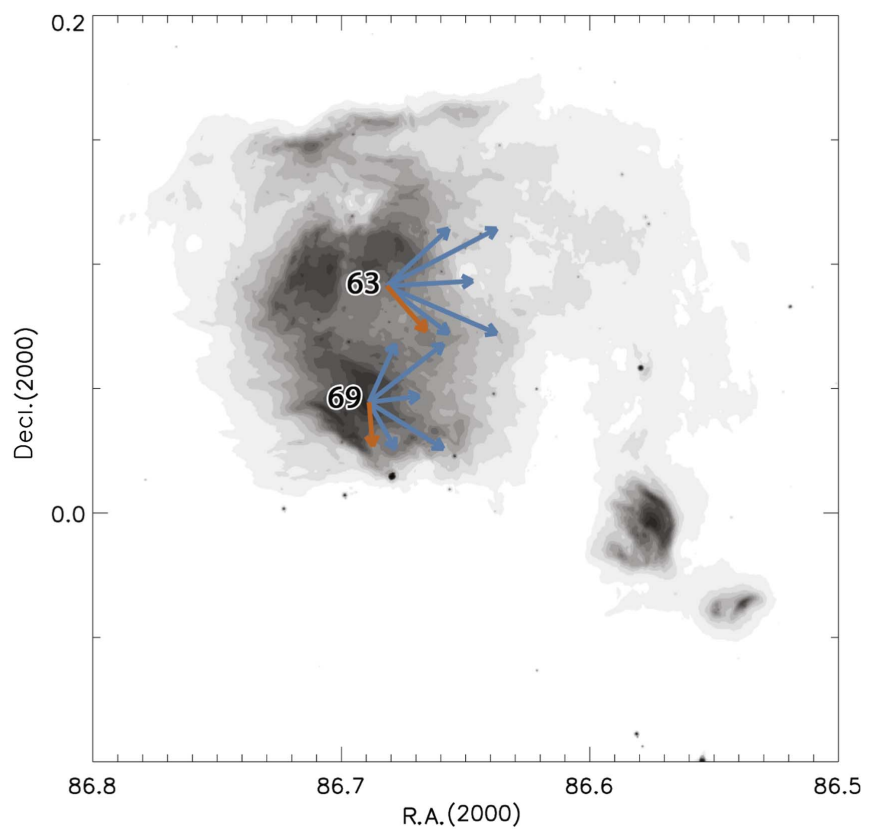

Figure 10. Same as Figure 8, but for NGC 2068.

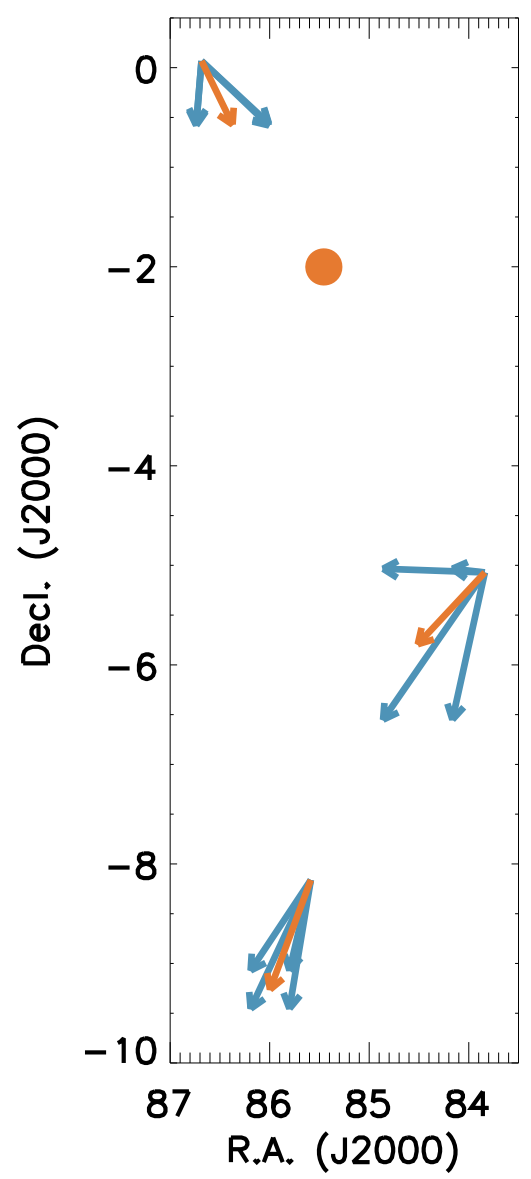

Figure 11. Orange arrows: 1sr proper motions of the regions of the Orion Complex. Blue arrows: $1 \sigma$ uncertainty range in these values. Orange dot shows the location of NGC 2024.

We acknowledge helpful discussions with Mark Reid, which led to testing the possible effects of phase delay in the ONC. G.N. O.-L., L.L., L.F.R., R.A.G.-L.L., G.P., and J.L.R. acknowledge
Table 8

Parameters of Elliptical Gaussian Model Fit for the Scattered Calibrators toward $\lambda$ Ori

\begin{tabular}{lcrcc}
\hline \hline Name & $\begin{array}{c}\nu \\
(\mathrm{GHz})\end{array}$ & \multicolumn{1}{c}{$\begin{array}{l}\theta_{\text {major }} \\
(\mathrm{mas})\end{array}$} & \multicolumn{1}{c}{$\begin{array}{l}\theta_{\text {minor }} \\
(\text { mas })\end{array}$} & $\begin{array}{c}\text { P.A. } \\
(\text { deg. })\end{array}$ \\
\hline J0536+0944 & 4.98 & $16.5 \pm 0.4$ & $9.9 \pm 0.1$ & $139.7 \pm 10.2$ \\
J0536+0944 & 8.42 & $5.0 \pm 0.1$ & $3.5 \pm 0.1$ & $150.3 \pm 1.7$ \\
J0532+0732 & 4.98 & $9.9 \pm 0.2$ & $7.2 \pm 0.1$ & $173.2 \pm 2.2$ \\
J0532+0732 & 8.42 & $4.3 \pm 0.1$ & $3.0 \pm 0.1$ & $174.0 \pm 1.2$ \\
J0544+1118 & 4.98 & $6.2 \pm 0.1$ & $5.1 \pm 0.1$ & $163.9 \pm 5.0$ \\
J0544+1118 & 8.42 & $2.9 \pm 0.1$ & $2.5 \pm 0.1$ & $7.3 \pm 2.0$ \\
\hline
\end{tabular}

DGAPA, UNAM, and CONACyT, Mexico for financial support. L.L. and G.O. also acknowledge support from von Humboldt Stiftung. N.J.E. was supported by NSF grant AST-1109116 to the University of Texas at Austin. P.A.B.G. acknowledges financial support from FAPESP. The National Radio Astronomy Observatory is operated by Associated Universities, Inc., under cooperative agreement with the NSF.

Software: AIPS (Greisen 2003), MPFIT (Markwardt 2009), Skyfield, Binary Star Combined Solution Package (Gudehus 2001).

\section{APPENDIX A $\lambda$ ORI SCATTERING}

As with all the other regions, $\lambda$ Ori was observed at $5 \mathrm{GHz}$. During the first epoch of the observations, J0536+0944 was used as a primary calibrator, which is located only 0.4 from the center of the cluster. Calibrating on this source could produce a coherent signal only on the baselines shorter than $1000 \mathrm{~km}$; that is, the baselines between Fort Davis, Kitt Peak, Los Alamos, Pie Town, and partially Owens Valley.

The second and third epochs were calibrated on J0532 +0732 , located 2.4 from the cluster center but still barely inside the $\lambda$ Ori ring. While this produced a significant improvement on the calibration over the first epoch, any baselines involving antennas at Hancock and St. Croix could not be calibrated. Since the longest baseline of VLBA was not used, uncertainties in source positions remained large. However, surprisingly, some baselines longer than the baselines involving these two antennas (including most baselines involving Maunakea) did produce some coherent signal.

To determine the cause of the poor signal and potentially find a primary calibrator that could cause an improvement on the data, in 2015 August we observed four calibrators-J0536 +0944 , J0532+0732, J0544+1118, and J0547+1223 - at both 5 and $8 \mathrm{GHz}$. J0547+1223 was known to produce good calibration; it is located almost $4^{\circ}$ from the cluster center. While it did appear as a point source at both wavelengths, such a large angular separation is larger than what is ideal for a primary calibrator. $\mathrm{J} 0544+1118$, located at 2.8 from the cluster center, had an appearance very similar to J0532+0732baselines involving $\mathrm{HN}$ and $\mathrm{SC}$ could not produce a coherent signal at $5 \mathrm{GHz}$. With the exception of J0547+1223, the other three calibrators showed a significant improvement at $8 \mathrm{GHz}$. However, even at this wavelength they did not appear like point sources. And even at $8 \mathrm{GHz}, \mathrm{J} 0536+0944$ did appear to be significantly poorer than any other calibrator.

The Gaussian model fits of the sizes of these sources is listed in Table 8 . These sizes are roughly consistent with $\lambda^{2}$. Because of this wavelength dependence, we believe that all radio 
observations of $\lambda$ Ori are significantly affected by the plasma scattering. A possible source of this scattering is the ionized gas in the $\lambda$ Ori ring that is currently $2.5-3^{\circ}$ in radius (Figure 1 ), left behind by an SN blast that originated 1 Myr ago (Dolan \& Mathieu 2002). However, it is surprising that $\lambda$ Ori is the only Orion region where scattering is a concern. Very few regions are known to be sources of significant plasma scattering processes that can be observed in this portion of radio regime; among them are the Galactic Center (Bower \& Backer 1998), NGC 6334 (Rodríguez et al. 2012), and Cygnus (Desai \& Fey 2001).

The fourth epoch of the observations was done at $8 \mathrm{GHz}$ and used $\mathrm{J} 0544+1118$ as the primary calibrator. Only two sources were detectable at a higher frequency (VLBA 85 and 87). After considering positional offsets that were introduced with several alterations of the primary calibrators, we determined that neither of these sources appear to be galactic. No further monitoring was done for the region.

One object of interest identified toward $\lambda$ Ori is VLBA 85. It has two components separated by 0.7 mas, possibly an AGN jet.

\section{APPENDIX B \\ COMMENTS ON THE INDIVIDUAL SOURCES IN THE ONC}

\section{B.1. Trapezium}

VLBA 5 (=GMR A) is found to be at a distance of $374 \pm 15 \mathrm{pc}$ with a proper motion of $\mu_{\alpha}=1.81 \pm 0.11 \mathrm{mas} \mathrm{yr}^{-1}$

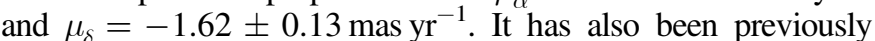
monitored with VLBA in a period from 2003 January to 2004 December by S07 and from 2006 September to 2007 March by MR. S07 found a distance solution to GMR A of $389_{-21}^{+24} \mathrm{pc}$ with $\mu_{\alpha}=1.89 \pm 0.12 \mathrm{mas} \mathrm{yr}^{-1}$ and $\mu_{\delta}=-1.67 \pm$ 0.19 mas yr $^{-1}$, which is consistent with the distance found in this work. On the other hand, MR found a distance solution of $418.4 \pm 18.2 \mathrm{pc}$ with $\mu_{\alpha}=1.82 \pm 0.09 \mathrm{mas} \mathrm{yr}^{-1}$ and $\mu_{\delta}=$ $-2.05 \pm 0.18$ mas yr$^{-1}$, a discrepancy on the order of $2 \sigma$ in distance and $\mu_{\delta}$. The difference in proper motion is unlikely to be attributed to a long-period multiplicity due to the lack of acceleration observed between S07 and this work. No information is available in regard to whether or not GMR A belongs to a compact binary.

VLBA 6 (=GMR F) is observed to be at a distance of $389 \pm$ $7 \mathrm{pc}$, with a proper motion of $\mu_{\alpha}=2.38 \pm 0.08 \mathrm{mas} \mathrm{yr}^{-1}$

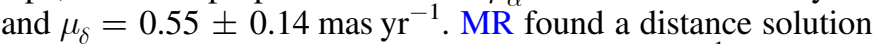
of $406.1 \pm 8.4 \mathrm{pc}$ with $\mu_{\alpha}=2.24 \pm 0.09 \mathrm{mas} \mathrm{yr}^{-1}$ and $\mu_{\delta}=$ $-0.66 \pm 0.18 \mathrm{mas} \mathrm{yr}^{-1}$. While the two distance estimates disagree, the proper motions are consistent, suggesting that GMR $F$ is unlikely to be a long-period binary. It has been identified as a double-lined spectroscopic binary by Prato et al. (2002) with $q \sim 0.31$, but no orbital solution is available.

VLBA 7 (=GMR H) is detected only in the first three epochs of the observations; therefore uncertainties in the solution are presented based only on the astrometric uncertainties and do not take into the account the systematic offsets. We obtain a distance solution of $372 \pm 15 \mathrm{pc}$ and $\mu_{\alpha}=2.22 \pm 0.18 \mathrm{mas} \mathrm{yr}^{-1}$ and $\mu_{\delta}=-3.08 \pm 0.55$. This was not observed by MR.

VLBA 8 (=GMR $G)$ is found to be at a distance of $377 \pm 5 \mathrm{pc}$ with $\mu_{\alpha}=3.82 \pm 0.10 \mathrm{mas} \mathrm{yr}^{-1}$ and $\mu_{\delta}=1.60 \pm$

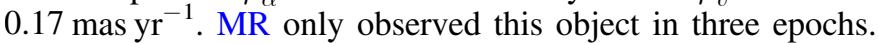
For this reason, they do not present a distance solution; however, the data taken by their program suggest a distance of
$382 \pm 4 \mathrm{pc}$, providing a good agreement to the distance obtained through in this work. However, proper motions obtained by MR are $\mu_{\alpha}=4.29 \pm 0.17 \operatorname{mas~yr}^{-1}$ and $\mu_{\delta}=$

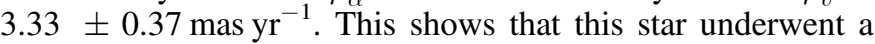
significant acceleration in the eight years between these observations, suggestive of a long-period binary; however, the magnitude of the acceleration is not sufficient to noticeably affect the proper motions during the $\sim$ two years covered by either program separately. No optical or IR companion to the system has been previously identified.

VLBA 9 (=GMR $25,=\theta^{1}$ Ori $\left.\mathrm{E}\right)$ is a known spectroscopic binary (Costero et al. 2008; Morales-Calderón et al. 2012) with a circular orbit, a period of 9.89520 days, $q \sim 1, i=74^{\circ}$, and masses of 2.807 and $2.797 M_{\odot}$. This was not previously observed by MR. We find a distance solution of $391 \pm 7 \mathrm{pc}$ with $\mu_{\alpha}=1.45 \pm 0.03 \mathrm{mas} \mathrm{yr}^{-1}$ and $\mu_{\delta}=1.02 \pm 0.08 \mathrm{mas} \mathrm{yr}^{-1}$, without taking into the account the orbital motion. The lack of eccentricity makes it difficult to constrain the position of the star in its orbit with the astrometric data, but the typical effect of this orbit on the distance solution is within $3 \mathrm{pc}$.

VLBA 11 (=GMR $12,=\theta^{1}$ Ori A) is a known triple system. One of the components has been detected through adaptive optics and Very Large Telescope Interferometer (VLTI) imaging (Close et al. 2013; Grellmann et al. 2013), with a projected separation of $\sim 0$ !' 2 or $\sim 70$ au. The other component is an eclipsing system that is also observed through spectroscopy with a period of 65.4 days (Stickland \& Lloyd 2000). Not accounting for the orbital motion, we obtain a distance solution of $380 \pm 7 \mathrm{pc}$ with $\mu_{\alpha}=4.81 \pm 0.07 \mathrm{mas} \mathrm{yr}^{-1}$ and $\mu_{\delta}=-2.33 \pm 0.09$ mas $\mathrm{yr}^{-1}$. Incorporating the orbital motion of the spectroscopic binary while solving for $i$ and $\Omega$ yields a comparable distance of $380 \pm 14 \mathrm{pc}$ with $\mu_{\alpha}=4.81 \pm$ $0.10 \mathrm{mas} \mathrm{yr}^{-1}, \mu_{\delta}=-2.53 \pm 0.12, \Omega=150 \pm 20^{\circ}$, and $i=$ $87 \pm 11^{\circ}$, therefore consistent with an eclipsing system. This result does not change significantly by assuming that the emission is coming from the secondary instead of the primary. MR found a distance toward GMR 12 of $417.9 \pm 9.2 \mathrm{pc}$ with $\mu_{\alpha}=4.82 \pm 0.09$ mas yr $^{-1}$ and $\mu_{\delta}=-1.54 \pm 0.18$ mas yr$^{-1}$. This solution did not take into account the orbital motion; however, including it does not significantly alter the fit. The distances between two observations are discrepant by $\sim 2 \sigma$. The difference in the measured proper motion is most likely driven by acceleration due to the orbital motion of the long-period binary.

Other confirmed galactic sources that have been detected toward the Trapezium include VLBA 13, 149, and 150, but as they have been detected in only two epochs, currently it is impossible to do a parallactic fit.

\section{B.2. Outside of the Trapezium}

VLBA 4/107 (=Brun 656) is located westward of the OMC $2 / 3$ filament. It was detected as an astrometric binary system, with VLBA 4 detected in epochs 1,4 , and 5 and VLBA 107 detected in epochs 2, 4, and 5. Orbital motion is clearly apparent in both stars. However, with only six positions it is impossible to fit all 13 parameters for both parallactic and orbital motion. Therefore we exclude $i$ and $\Omega$ from the fit, assuming a face-on orientation. In the follow-up work with additional data it would be possible to present a full solution. Potentially a member of a triple system as Köhler et al. (2006) identify an additional companion 0 ". 4 or $\sim 150$ au away, this could mean that VLBA 4/107 are members of a triple system. 
This should have little impact on the astrometry after a twoyear baseline. We obtain a distance solution of $369 \pm 10 \mathrm{pc}$ with the proper motions of the compact system of $\mu_{\alpha}=2.36 \pm$ $0.69 \mathrm{mas} \mathrm{yr}^{-1}$ and $\mu_{\delta}=0.06 \pm 1.05 \mathrm{mas} \mathrm{yr}^{-1}$, and the scatter and the dependence between these parameters is shown in Figure 3 . This proper motion is most likely not representative of the true proper motion of the triple system. We find the period of the compact system to be $6.27 \pm 0.54$ years and an $M \cos ^{3} i$ of both components of $1.70 \pm 0.16$ and $1.62 \pm 0.38$ $M_{\odot}$. The spectral type for the primary has been previously reported to be G2III (Hillenbrand 1997).

VLBA 14 (=V1699 Ori) is located toward NGC 1977. We find a distance solution of $401 \pm 7 \mathrm{pc}$ with $\mu_{\alpha}=1.76 \pm$ $0.05 \mathrm{mas} \mathrm{yr}^{-1}$ and $\mu_{\delta}=-0.89 \pm 0.16$ mas yr$^{-1}$.

VLBA 16 (=Parenago 1469) is located westward of the Trapezium. It was detected only in epochs 1,2 , and 4 . We find a distance solution of $394 \pm 4 \mathrm{pc}$ with $\mu_{\alpha}=-7.22 \pm$ $0.06 \mathrm{mas} \mathrm{yr}^{-1}$ and $\mu_{\delta}=-0.99 \pm 0.08 \mathrm{mas} \mathrm{yr}^{-1}$. Proper motions for this star are uncommonly large and appear to be projected from the Trapezium cluster (see Section 4.3).

VLBA 18 (=Parenago 1724) is located westward of the OMC $2 / 3$ filament. We find a distance solution of $398 \pm 7 \mathrm{pc}$ with $\mu_{\alpha}=0.06 \pm 0.20$ mas yr$^{-1}$ and $\mu_{\delta}=6.95 \pm 0.15 \mathrm{mas} \mathrm{yr}^{-1}$. Similarly to VLBA 16, VLBA 18 also has a very large proper motion vector, which projects back to the center of the Trapezium cluster. It was previously identified by Neuhaeuser et al. (1998) as a runaway star.

VLBA 19 (=Parenago 1540) is located westward of the Trapezium. It is a known double-lined spectroscopic binary (Marschall \& Mathieu 1988). Without accounting for the orbital motion, we obtain a distance solution of $404 \pm 11 \mathrm{pc}$ with $\mu_{\alpha}=-3.88 \pm 0.13$ mas yr $^{-1}$ and $\mu_{\delta}=-1.10 \pm$ 0.15 mas yr $^{-1}$. Incorporating an orbital fit and solving for $i$ and $\Omega$ yields a distance of $386 \pm 7 \mathrm{pc}$ with

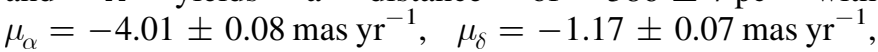
$i=104 \pm 12^{\circ}$, and $\Omega=69 \pm 11^{\circ}$. This results in the masses of the components of $0.49 \pm 0.10$ and $0.37 \pm 0.07 M_{\odot}$; however, the spectral types of $\mathrm{K} 3 \mathrm{~V}$ and $\mathrm{K} 5 \mathrm{~V}$ cause these masses to be somewhat suspect. With an assumption that the emission is coming from the secondary with thew rotated by $180^{\circ}$, an alternate family of solutions is found at $413 \pm$ $12 \mathrm{pc} \quad$ with $\mu_{\alpha}=-3.85 \pm 0.14 \mathrm{mas} \mathrm{yr}^{-1}, \quad \mu_{\delta}=-1.12 \pm$ $0.08 \mathrm{mas} \mathrm{yr}^{-1}, \quad i=56 \pm 12^{\circ}, \quad$ and $\Omega=130 \pm 14^{\circ}$, and masses of $0.78 \pm 0.40$ and $0.60 \pm 0.28 M_{\odot}$. Similarly to VLBA 16 and 18, this system has a very large proper motion vector that projects back to the Trapezium cluster, and it has been previously theorized to be a runaway star by Marschall \& Mathieu (1988).

VLBA 22 (=HD 37150) is located toward the south-east of the Trapezium. We obtain a distance solution of $394 \pm 7 \mathrm{pc}$ with $\mu_{\alpha}=1.32 \pm 0.05$ mas yr$^{-1}, \mu_{\delta}=0.56 \pm 0.12$ mas yr $^{-1}$.

VLBA 27/28 (=NU Ori) shows considerable motion, but it cannot be fitted yet due to multiplicity. VLBA 27 has been detected in epochs 1, 3, and 5, and VLBA 28 has been detected in epoch 1, with the projected separation of 35 mas from VLBA 27. A single point-source was detected in epoch 4, tentatively interpreted to be associated with VLBA $28, \sim 8$ mas away from the expected position of VLBA 27. This companion system has been previously predicted to exist by Grellmann et al. (2013) based on the VLTI observations. In addition, this system contains a known spectroscopic binary with a period of 19 days and a $a_{1} \sin i$ of $\sim 0.15$ mas (Abt et al. 1991); a hint of extension is seen toward VLBA 27 in some epochs, and this could be the source. This system also has a wider companion with a separation of $\sim 0$. 5 (Köhler et al. 2006). Given the fact that the primary was detected only in the spring epochs without any fall epochs, we cannot provide even rough constraints on its distance. Follow-up monitoring would be needed in order to accurately understand the motions of this system.

VLBA 34 (=HD 37017) is a known double-lined spectroscopic binary (Bolton et al. 1998). Without orbital motion, we obtain a distance solution of $360 \pm 7 \mathrm{pc}$ with $\mu_{\alpha}=1.87 \pm$ $0.07 \mathrm{mas} \mathrm{yr}^{-1}, \mu_{\delta}=1.17 \pm 0.24 \mathrm{mas} \mathrm{yr}^{-1}$. Solving for $i$ and $\Omega$, we obtain two possible results due to the lack of constraints in the direction of the orbit. These results are $i=53 \pm 23^{\circ}$ and $i=127 \pm 28^{\circ}$, both with $\Omega=131 \pm 26^{\circ}$ and the distance solution of $378 \pm 10 \mathrm{pc}$ with $\mu_{\alpha}=1.88 \pm 0.09 \mathrm{mas} \mathrm{yr}^{-1}$, $\mu_{\delta}=1.20 \pm 0.14 \mathrm{mas} \mathrm{yr}^{-1}$. This corresponds to masses of the components of $4.09 \pm 4.41$ and $2.14 \pm 2.22 M_{\odot}$. Assuming that the emission is coming from the secondary does not change the solution significantly; the estimated distance becomes $383 \pm 5 \mathrm{pc}$, although the inclination angle becomes $76 \pm 18^{\circ}$.

VLBA 105 (=Parenago 2148) is located toward the OMC 2/ 3 filament. It was detected only in epochs 2,3 , and 5 . The positional uncertainties, particularly in epoch 2 , are rather substantial as the source appears to be marginally extended in $\alpha$. Without accounting for any of the systematic offsets, we find a distance solution of $388 \pm 53 \mathrm{pc}$ with $\mu_{\alpha}=0.33 \pm$ $0.05 \mathrm{mas} \mathrm{yr}^{-1}, \mu_{\delta}=-1.34 \pm 0.43$ mas yr $^{-1}$.

VLBA 114 (=Parenago 1778) is located toward NGC 1977. It was not detected in epoch 1 . We obtain a distance solution of $437 \pm 83 \mathrm{pc}$ with $\mu_{\alpha}=2.54 \pm 0.30$ mas yr $^{-1}, \mu_{\delta}=-1.30 \pm$ 0.64 mas $\mathrm{yr}^{-1}$. The fit is rather poor, despite the substantial positional uncertainties. This could be attributed to acceleration due to multiplicity.

Other galactic sources identified in the region that were detected only in two epochs are VLBA 33, 110, and 116. VLBA 115 has also been detected in two epochs, and although the $\alpha$ offset is consistent with belonging to the ONC (2.7 mas), the $\delta$ offset is over 13 mas; this is likely due to multiplicity.

\section{APPENDIX C COMMENTS ON THE REMAINING REGIONS}

\section{C.1. $L 1641$}

Only two galactic sources, VLBA 45 and 46, have been detected toward L1641. Both of them are located on the southern end of the cloud, at $\delta<-8^{\circ}$. The solutions toward them are somewhat more uncertain than they are toward sources located within the ONC, with very large uncertainties and imprecise fit. Preliminary fits result in distance solutions of $424 \pm 12 \mathrm{pc}$ with $\mu_{\alpha}=0.68 \pm 0.06$ mas yr $^{-1}, \mu_{\delta}=-0.31 \pm 0.22$ mas yr $^{-1}$ for VLBA 45 and $433 \pm 28$ pc with $\mu_{\alpha}=0.25 \pm 0.04$ mas yr $^{-1}$, $\mu_{\delta}=-0.47 \pm 0.25 \mathrm{mas} \mathrm{yr}^{-1}$ for VLBA 46. The fit appears to be somewhat dubious, and the observed positions do not agree with the best fits for both sources in epochs 1 and 4. It is possible that this offset is attributable to multiplicity in both of these objects, although the fact that the magnitude of the offset is comparable for both sources at each epoch makes it more dubious. Therefore we treat this offset as the pointing error due to calibration and solve for a common offset for both sources.

We correct the positions of the first epoch by $\Delta \alpha=$ 0.256 mas and $\Delta \delta=0.771$ mas and the positions of the fourth 
epoch by $\Delta \alpha=0.204$ mas and $\Delta \delta=0.659$ mas. It is somewhat curious that this offset is comparable in both epochs. After this correction, the distance solution becomes $425 \pm 12 \mathrm{pc}$ with $\mu_{\alpha}=0.68 \pm 0.09$ mas yr $^{-1}, \mu_{\delta}=-0.51 \pm 0.25 \mathrm{mas} \mathrm{yr}^{-1}$ for VLBA 45 and $431 \pm 13 \mathrm{pc}$ with $\mu_{\alpha}=0.13 \pm$ 0.25 mas yr$^{-1}, \mu_{\delta}=-1.05 \pm 0.18$ mas yr$^{-1}$ for VLBA 46 . This offset has a very small overall effect on the distance, but the proper motions are somewhat uncertain.

In addition to the galactic sources, a number of extragalactic sources can be of interest. VLBA 39/40, 41/42, 47/48, 89/90, and 94/95/96 appear to exhibit no motion between epochs, but they appear to be extended, double, or even triple objects. They can probably be attributable to the AGN jets. It is curious that so many of these extended sources appear to be in the direction of this particular region.

\section{C.2. NGC 2068}

The second epoch of the observations of this region was strongly affected by a pointing error, and this offset is also present in nearby sources that can otherwise be considered extragalactic. A possible explanation is that one of the secondary calibrators had an extremely weak detection in this epoch. We solve for a common offset for all sources of $\Delta \alpha=-0.559$ mas, $\Delta \delta=-0.515$ mas.

Three galactic sources have been detected toward NGC 2068: VLBA 63, 68 (=HD 290862), and 69. After removing the offset, we obtain a distance solution of $383 \pm 7 \mathrm{pc}$ with $\mu_{\alpha}=-1.02 \pm$ 0.10 mas yr $^{-1}, \mu_{\delta}=-0.52 \pm 0.15$ mas yr $^{-1}$ for VLBA 63 and $392 \pm 5 \mathrm{pc}$ with $\mu_{\alpha}=-0.01 \pm 0.10$ mas yr $^{-1}, \mu_{\delta}=-0.49 \pm$ 0.08 mas yr $^{-1}$ for VLBA 69. However, we can offer only an extremely noisy tentative solution for VLBA 68 of $455 \pm 113 \mathrm{pc}$ with $\mu_{\alpha}=0.35 \pm 0.27$ mas yr$^{-1}, \mu_{\delta}=0.83 \pm 0.83$ mas yr$^{-1}$, the reason being that VLBA 68 appears to be a multiple system (although no companion has been directly detected), which greatly affects the positions. At this time we cannot perform an orbital fit for the system.

\section{C.3. NGC 2024}

VLBA $61 / 62$ has been identified as an astrometric binary, with the first component present in all five epochs, while the second component is detected only in epochs 1,4 , and 5 . We find a distance to the system of $434 \pm 10 \mathrm{pc}$ with $\mu_{\alpha}=0.47 \pm 0.32$ mas yr$^{-1}, \mu_{\delta}=0.39 \pm 0.62 \mathrm{mas} \mathrm{yr}^{-1}$, and the scatter and the dependence between these parameters is shown in Figure 3. The period of the binary is $9.50 \pm 0.67 \mathrm{yr}$, with an inclination of $141 \pm 6^{\circ}$ and masses of both components of $1.85 \pm 0.58$ and $0.95 \pm 0.22 M_{\odot}$. Unfortunately, this system has not been detected at any other wavelength regime than in radio; therefore a comparison of masses to spectral types is impossible. While the orbital fit itself is convergent, it must be noted that some uncertainty does remain due to the limited number of detections, and although unlikely, a possibility of a somewhat larger distance of $\sim 444 \mathrm{pc}$ as well as a somewhat steeper inclination angle cannot be ruled out.

VLBA 58 is another star that was detected in all five epochs in this region, and it also appears to be a binary due to its peculiar motions from one epoch to the next. At this time, an orbit to it cannot be fitted, but we obtain a very tentative solution of $449 \pm 24 \mathrm{pc}$ with $\mu_{\alpha}=0.04 \pm 0.31 \mathrm{mas} \mathrm{yr}^{-1}$,

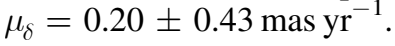

Three other stellar objects have been detected toward the region, but only in three epochs; therefore solutions are somewhat uncertain as they do not take into account any systematic offsets. VLBA 148 has a distance solution of $412 \pm 5 \mathrm{pc}$ with $\mu_{\alpha}=0.19 \pm 0.44$ mas yr $^{-1}, \quad \mu_{\delta}=-0.97 \pm 0.27$ mas yr $^{-1}$. VLBA 125 is presently found at a distance of $536 \pm 30$ with $\mu_{\alpha}=-0.43 \pm 0.16$ mas yr $^{-1}, \mu_{\delta}=1.03 \pm 0.42$ mas yr $^{-1}$; this solution is rather tentative due to the astrometric errors and quality of the fit. However, VLBA 126 has a measured distance of $356 \pm 4 \mathrm{pc}$ with $\mu_{\alpha}=0.55 \pm 0.10 \operatorname{mas~yr}^{-1}, \mu_{\delta}=-0.10 \pm$ 0.15 mas yr $^{-1}$.

It is clear that VLBA 125 and 126 have a measured distance that is decidedly different from what is found toward other objects in the region. A possible explanation is that these stars may belong to an as-yet unseen binary system. Whether this could also be the case for VLBA 148 is unclear.

Other galactic sources identified toward NGC 2024 are VLBA 124 and 153, although only two epochs are currently available.

A number of extragalactic objects of interest have also been identified. VLBA 56 shows a clear signature of an AGN jet. VLBA 146/147 has two components, as well as a probable extragalactic jet. While previously VLBA 55 was identified as a Class II YSO based on its IR colors, it shows no positional offset between epochs. It was notable for being extremely bright in radio (the highest VLBA flux of $338 \mathrm{mJy}$ at $5 \mathrm{GHz}$ ).

\section{C.4. $\sigma$ Ori}

Only one Galactic object has been identified toward $\sigma$ Ori among those detected with VLBA. VLBA 145 (=HD 294300) was not monitored in epochs 1 and 2; therefore currently only three epochs of astrometry are available. VLBA 145 is found at a distance of $302 \pm 32 \mathrm{pc}$ with $\mu_{\alpha}=-4.92 \pm 0.66$ mas yr$^{-1}$, $\mu_{\delta}=4.67 \pm 1.37 \mathrm{mas} \mathrm{yr}^{-1}$. Whether this distance solution is trustworthy or not still remains to be tested; while $\sigma$ Ori is most likely spatially separate from NGC 2024, a difference in distance of over $100 \mathrm{pc}$ would be surprising. Sherry et al. (2008) previously estimated the distance toward $\sigma$ Ori based on main-sequence fitting to be $420 \pm 30 \mathrm{pc}$, quite close to the distances we obtain to NGC 2024 members. Combined with the rather high proper motions for VLBA 145 of $9.7 \mathrm{~km} \mathrm{~s}^{-1}$ and a somewhat poor fit, these findings could imply that this star belongs to a binary system; therefore further monitoring would be needed to better understand the kinematics of the system.

\section{C.5. $L 1622$}

In the VLA survey, only two sources have been identified toward L1622: one was a known YSO, and one did not have any classification. The former one was not detected with VLBA, and the latter (VLBA 84) did not exhibit any positional offset between observations. No parallax toward this region can be measured. No further monitoring was done past epoch 2 .

\section{REFERENCES}

Abt, H. A., Wang, R., \& Cardona, O. 1991, ApJ, 367, 155

Alcalá, J. M., Covino, E., Torres, G., et al. 2000, A\&A, 353, 186

Alves, J., \& Bouy, H. 2012, A\&A, 547, A97

Bally, J. 2008, in Handbook of Star Forming Regions, Vol. 1, ed. B. Reipurth (San Fransisco, CA: ASP), 459

Bally, J., Langer, W. D., Stark, A. A., \& Wilson, R. W. 1987, ApJL, 312, L45 
Bolton, C. T., Harmanec, P., Lyons, R. W., Odell, A. P., \& Pyper, D. M. 1998, A\&A, 337, 183

Bouy, H., Alves, J., Bertin, E., Sarro, L. M., \& Barrado, D. 2014, A\&A, 564, A29

Bower, G. C., \& Backer, D. C. 1998, ApJL, 496, L97

Close, L. M., Males, J. R., Morzinski, K., et al. 2013, ApJ, 774, 94

Costero, R., Allen, C., Echevarría, J., et al. 2008, RMxAC, 34, 102

Da Rio, N., Robberto, M., Soderblom, D. R., et al. 2010, ApJ, 722, 1092

Da Rio, N., Tan, J. C., Covey, K. R., et al. 2016, ApJ, 818, 59

de Bruijne, J. H. J., Rygl, K. L. J., \& Antoja, T. 2014, EAS Publ. Ser., 67, 23

de Zeeuw, T., Hoogerwerf, R., \& de Bruijne, J. 2001, in ASP Conf. Ser. 228,

Dynamics of Star Clusters and the Milky Way, ed. S. Deiters et al. (San

Francisco, CA: ASP), 201

Desai, K. M., \& Fey, A. L. 2001, ApJS, 133, 395

Dolan, C. J., \& Mathieu, R. D. 2002, AJ, 123, 387

Duchêne, G., \& Kraus, A. 2013, ARA\&A, 51, 269

Duquennoy, A., \& Mayor, M. 1991, A\&A, 248, 485

Fischer, D. A., \& Marcy, G. W. 1992, ApJ, 396, 178

Genzel, R., Reid, M. J., Moran, J. M., \& Downes, D. 1981, ApJ, 244, 884

Goddi, C., Humphreys, E. M. L., Greenhill, L. J., Chandler, C. J., \& Matthews, L. D. 2011, ApJ, 728, 15

Gómez, L., Rodríguez, L. F., Loinard, L., et al. 2008, ApJ, 685, 333

Greisen, E. W. 2003, in Information Handling in Astronomy: Historical Vistas, Vol. 285, ed. A. Heck (New York: Springer), 109

Grellmann, R., Preibisch, T., Ratzka, T., et al. 2013, A\&A, 550, A82

Gualandris, A., Portegies Zwart, S., \& Eggleton, P. P. 2004, MNRAS, 350, 615

Gudehus, D. H. 2001, BAAS, 33, 850

Gutermuth, R. A., Pipher, J. L., Megeath, S. T., et al. 2011, ApJ, 739, 84

Hartmann, L. 2001, AJ, 121, 1030

Hillenbrand, L. A. 1997, AJ, 113, 1733

Hillenbrand, L. A., \& Hartmann, L. W. 1998, ApJ, 492, 540

Hirota, T., Bushimata, T., Choi, Y. K., et al. 2007, PASJ, 59, 897

Houk, N., \& Swift, C. 1999, University of Michigan Catalogue of Twodimensional Spectral Types for the HD Stars, Vol 5. (Ann Arbor, MI: Univ. Michigan)

Hsu, W.-H., Hartmann, L., Allen, L., et al. 2013, ApJ, 764, 114

Jeffries, R. D. 2007, MNRAS, 376, 1109

Kapteyn, J. C. 1918, ApJ, 47, 104

Kim, M. K., Hirota, T., Honma, M., et al. 2008, PASJ, 60, 991

Köhler, R., Petr-Gotzens, M. G., McCaughrean, M. J., et al. 2006, A\&A, 458, 461

Kounkel, M., Hartmann, L., Loinard, L., et al. 2014, ApJ, 790, 49

Kounkel, M., Hartmann, L., Tobin, J. J., et al. 2016, ApJ, 821, 8
Kraus, S., Weigelt, G., Balega, Y. Y., et al. 2009, A\&A, 497, 195

Loinard, L., Mioduszewski, A. J., Torres, R. M., et al. 2011, RMxAC, 40, 205

Lombardi, M., Alves, J., \& Lada, C. J. 2011, A\&A, 535, A16

Markwardt, C. B. 2009, in ASP Conf. Ser. 411, Astronomical Data Analysis Software and Systems XVIII, ed. D. A. Bohlender, D. Durand, \& P. Dowler (San Francisco, CA: ASP), 251

Marschall, L. A., \& Mathieu, R. D. 1988, AJ, 96, 1956

Megeath, S. T., Gutermuth, R., Muzerolle, J., et al. 2012, AJ, 144, 192

Melis, C., Reid, M. J., Mioduszewski, A. J., Stauffer, J. R., \& Bower, G. C. 2014, Sci, 345, 1029

Menten, K. M., Reid, M. J., Forbrich, J., \& Brunthaler, A. 2007, A\&A, 474,515

Morales-Calderón, M., Stauffer, J. R., Stassun, K. G., et al. 2012, ApJ, 753, 149

Muench, A., Getman, K., Hillenbrand, L., \& Preibisch, T. 2008, in Handbook of Star Forming Regions, Vol. 1, ed. B. Reipurth (San Fransisco, CA ASP), 483

Neuhaeuser, R., Wolk, S. J., Torres, G., et al. 1998, A\&A, 334, 873

Nishimura, A., Tokuda, K., Kimura, K., et al. 2015, ApJS, 216, 18

Ochsendorf, B. B., Brown, A. G. A., Bally, J., \& Tielens, A. G. G. M. 2015, ApJ, 808, 111

O’Dell, C. R., Poveda, A., Allen, C., \& Robberto, M. 2005, ApJL, 633, L45

Ortiz-León, G. N., Loinard, L., Dzib, S. A., et al. 2017a, ApJ, 834, 141

Ortiz-León, G. N., Loinard, L., Kounkel, M., et al. 2017b, ApJ, 834, 143

Pickering, W. H. 1917, HarCi, 205, 1

Poveda, A., Allen, C., \& Hernández-Alcántara, A. 2005, ApJL, 627, L61

Prato, L., Simon, M., Mazeh, T., et al. 2002, ApJ, 569, 863

Raghavan, D., McAlister, H. A., Henry, T. J., et al. 2010, ApJS, 190, 1

Reid, M. J., \& Brunthaler, A. 2004, ApJ, 616, 872

Reid, M. J., \& Honma, M. 2014, ARA\&A, 52, 339

Rodríguez, L. F., Gómez, Y., \& Tafoya, D. 2012, MNRAS, 420, 279

Sandstrom, K. M., Peek, J. E. G., Bower, G. C., Bolatto, A. D., \& Plambeck, R. L. 2007, ApJ, 667, 1161

Schlafly, E. F., Green, G., Finkbeiner, D. P., et al. 2014, ApJ, 786, 29

Seidelmann, P. K. 1992, Explanatory Supplement to the Astronomical Almanac (Mill Valley, CA: Univ. Science Books)

Sherry, W. H., Walter, F. M., Wolk, S. J., \& Adams, N. R. 2008, AJ, 135, 1616 Stassun, K. G., Mathieu, R. D., Vaz, L. P. R., Stroud, N., \& Vrba, F. J. 2004, ApJS, 151, 357

Stickland, D. J., \& Lloyd, C. 2000, Obs, 120, 141

Strassmeier, K. G. 2009, A\&ARv, 17, 251

Strom, K. M., Strom, S. E., Carrasco, L., \& Vrba, F. J. 1975, ApJ, 196, 489 